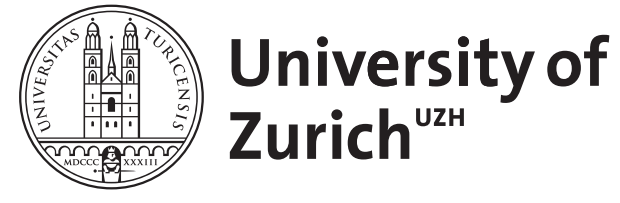

\title{
Der Beschluss als Organakt
}

\author{
Ernst, Wolfgang
}

Posted at the Zurich Open Repository and Archive, University of Zurich ZORA URL: https://doi.org/10.5167/uzh-71248

Book Section

Published Version

Originally published at:

Ernst, Wolfgang (2012). Der Beschluss als Organakt. In: Häublein, Martin. Rechtsgeschäft, Methodenlehre und darüber hinaus : Liber amicorum für Detlef Leenen zum 70. Geburtstag am 4. August 2012. Berlin: de Gruyter, 1-42. 


\title{
Der Beschluss als Organakt
}

\author{
WOLFGANG ERNST
}

\section{Die Vorstellung vom Beschluss als mehrseitigem Rechtsgeschäft}

Über den Beschluss heisst es bei Andreas von Tubr, er sei ein mehrseitiges Rechtsgeschäft. Er entstehe aus einer Mehrheit einzelner Willenserklärungen. ${ }^{1}$ Als diese sah von Tubr die einzelnen Stimmabgaben an. Der Beschluss sei ein „Zusammenwirken mehrerer inhaltlich übereinstimmender Willenserklärungen, also ein mehrseitiges Rechtsgeschäft" ${ }^{2}$ Der Beschluss soll danach durch die einzelnen, zustimmenden Stimmabgaben gegeben sein, ähnlich wie das Rechtsgeschäft „Vertrag“ durch die beiden Willenserklärungen Angebot und Annahme gegeben ist. Die Stimmabgabe des einzelnen Mitglieds sei jeweils „ein Stück des Rechtsgeschäfts, an dem die übrigen Abstimmenden mitbeteiligt sind“. ${ }^{3}$ Auch von einer „Vereinigung der Stimmen zum Beschluss" ist in diesem Zusammenhang die Rede. ${ }^{4}$ Man könnte auch sagen, die Stimmabgabe sei ein Teil, der Beschluss das Ganze desselben Rechtsgeschäfts. Nach dieser Vorstellung ist der Beschluss ein Rechtsgeschäft, das nur von den Zustimmenden (der Mehrheit) herrührt; diese zusammen sind auctor des Beschlusses. Hingegen tragen die Nichtzustimmenden zum so verstandenen Rechtsakt Beschluss gar nichts bei; ihre Stimmabgaben werden nicht Bestandteil des Rechtsgeschäfts „Beschluss“. Von den Gegenstimmen und Enthaltungen ihrer Stimmabgaben kann man nur sagen, dass sie die Fassung des Beschlusses nicht haben verhindern können; mit der mehrheitlichen Beschlussfassung haben sie sich daher erledigt, während die JA-Stimmen im Beschluss fortwirken. Nach dieser Vorstellung stehen die Nichtzustimmenden dem abgeschlossenen Rechtsgeschäft „Beschluss“ als extranei gegenüber, ebenso wie die bei der Abstimmung Abwesenden. Den Umstand, dass auch diese Mitglieder durch den Beschluss gebunden sind, muss man als eine Art Drittwirkung des Beschlusses verstehen, die sich dem Mehrheitsprinzip verdankt: Der Beschluss erscheint so als ein mehrseitiges Rechtsgeschäft nur der

${ }^{1}$ Der Allgemeine Teil des Deutschen Bürgerlichen Rechts, Bd. II/1, 1914, S. 232.

${ }^{2}$ A. von Tuhr, Der Allgemeine Teil des Deutschen Bürgerlichen Rechts, Bd.I, 1910, S. 514

3 von Tubr (o. Fn. 2), S. 516.

${ }^{4}$ R. Ruth, ZHR 88 (1926), 454, 526. 
Zustimmenden, an welches die Nichtzustimmenden als extranei wegen des Mehrheitsprinzips gebunden sind. So bildete für von Tubr das Mehrheitsprinzip die differentia specifica, durch die sich der Beschluss von anderen mehrseitigen Rechtsgeschäften unterscheiden sollte. In seinem Allgemeinen Teil des Schweizerischen Obligationenrechts heißt es: „Der Beschluss erfordert in der Regel keine Einigung (welche beim Vertrag wesentlich ist), sondern nur Stimmenmehrheit: Der Wille der Mehrheit ist auch für die überstimmten und für die abwesenden Beteiligten maßgebend. " 5 Der Grund für die Bindung an den Beschluss erscheint daher in eigentümlicher Weise abhängig vom Stimmverhalten: Wer dem Beschluss zugestimmt hat, ist gebunden, weil dies seinem rechtsgeschäftlich betätigten Willen entspricht; die Überstimmten und die Abwesenden sind gebunden wegen des Mehrheitsprinzips.

Weil von Tubr in dieser, im Mehrheitsprinzip begründeten Wirkung auf die extranei geradezu das begriffsbestimmende Merkmal des Beschlusses sah, ${ }^{6}$ gelangten diejenigen, die ihm in diesem Verständnis nachgefolgt sind, zu der Frage, ob eine Versammlungsentscheidung, für die Einstimmigkeit erforderlich ist, überhaupt als Beschluss bezeichnet werden kann; derselbe merkwürdige Zweifel entstand hinsichtlich der Entschließung durch einen Alleingesellschafter: ${ }^{7}$ In diesen Fällen fehlt ja die für charakteristisch gehaltene Fremdwirkung der mehrheitlichen JA-Stimmen auf diejenigen, die nicht für den Antrag gestimmt haben. Schließlich ergab und ergibt sich aus dem Ansatz von Tubrs, dass die Feststellung (Verlautbarung, Eröffnung) des Beschlusses nur noch ein bloß deklaratorischer Akt sein kann (und insofern entbehrlich ist), da der Beschluss als Rechtsgeschäft bereits mit der letzten Stimmabgabe vollendet sein soll. Weitgehend war damit der Beschluss in die Rechtsgeschäftslehre integriert, dies in einem gewissen Gegensatz zu Otto von Gierke, dessen Lehre vom „Gemeinwillen“ den Beschluss vom gewöhnlichen Rechtsgeschäft abgerückt hatte. ${ }^{8}$

Hans Erich Feine sah im Beschluss einen einheitlichen „Gesamtwillensakt“, zu dem sich die Stimmabgaben „verschmelzen“; in diesem Vorgang sollen die einzelnen Stimmabgaben „untergehen“, indem sie von dem einheitlichen Beschluss „absorbiert“ werden. ${ }^{9}$ Im Unterschied zur Vorstellung

${ }^{5}$ A. von Tubr, Allg. Teil des Schweiz. Obligationenrechts, 1. Halbbd., 1924, S. 122; s. auch von Tubr (o. Fn. 1), S. 235 f.

${ }^{6}$ Ausdrücklich ebenso D. Medicus, Allgemeiner Teil des BGB, 10. Aufl. 2010, Rn. 205.

7 Siehe die charakteristischen Erörterungen bei J. Baltzer, Der Beschluss als rechtstechnisches Mittel organschaftlicher Funktion im Privatrecht, 1965, S. 35 f.; für die Ausgrenzung der durch eine Person herbeigeführten Entschliessung aus dem Beschlussbegriff auch W. Zöllner, Festschrift Lutter, 2000, S. 821, 822.

8 O. v. Gierke, Deutsches Privatrecht, Bd. I, 1895, S. 283 Fn. 2 a.E.; s. auch S. 501 ff.

${ }^{9}$ H. E. Feine, in: Ehrenbergs Handbuch des Handelsrechts, 3. Bd./II. Abt., 1929, S. 517. 
von Tubrs besteht nach Feine der Beschluss nicht in den positiven Stimmabgaben; diese bewirken aber nach seiner Vorstellung den Beschluss, um in diesem Wirkakt gleichsam zu vergehen. ${ }^{10}$ Eine ähnliche Abschichtung des Beschlusses von den Stimmabgaben klingt auch bei Karsten Schmidt an, wenn er meint: „Der Beschluss gewinnt seine Wirksamkeit aus Willenserklärungen (den abgegebenen Stimmen), ist aber selbst keine Willenserklärung. “11 Während bei von Tubr das Verhältnis von positiver Stimmabgabe und Beschluss als das Verhältnis vom Teil zum Ganzen angesehen werden kann, scheint es sich bei Feine und Schmidt eher um ein Verhältnis von Ursache und Wirkung (vorher/nachher) zu handeln. Aber auch für Feine und Schmidt ist der Beschluss eine unmittelbare Wirkung der Stimmabgaben, wird er durch diese hervorgebracht.

Die Ansicht vom Beschluss als mehrseitigem, aus den Stimmabgaben zusammengefügtem Rechtsgeschäft bestimmt auch das heute maßgebliche Schrifttum. So hieß es in der grundlegenden Studie Baltzers, der Beschluss sei als mehrseitiges Rechtsgeschäft anzusehen, da er stets aus mehreren Willenserklärungen entstehe; die Einzelstimme sei „Entstehungselement“ des Beschlusses. ${ }^{12}$ Auch in einer jüngeren Studie zur Haftung für Gremienentscheidungen wird dieses Verständnis von Beschluss und Stimmabgabe zugrunde gelegt. ${ }^{13}$ In Werner Flumes Werk zur Juristischen Person heißt es unter Berufung u.a. auf von Tubr: „Die einzelne Stimmabgabe ist eine Willenserklärung, und der Beschluss ist als Zusammenwirken mehrerer inhaltlich übereinstimmender Willenserklärungen ein mehrseitiges Rechtsgeschäft. “14 In der Darstellung durch Medicus ${ }^{15}$ wird hervorgehoben, dass die den Beschluss erzeugenden Willenserklärungen gleichlautend seien. Weil danach nur die zustimmenden Erklärungen den gefassten Beschluss ausmachen, müssen auch nach der Formel von Medicus diejenigen, die anders gestimmt hatten als mit JA, hinsichtlich des aus der Abstimmung hervorgegangenen Rechtsgeschäfts „Beschluss“ extranei sein. Auf die „an der Beschlussfassung beteiligten Per-

10 Hiergegen wieder Baltzer (o. Fn. 7), S. 141 f., 170.

11 K. Schmidt, Gesellschaftsrecht, 4. Aufl. 2002, S. 436; ähnl. F. Jacoby, Das private Amt, 2007, S. 421: „Beschlussfassung beruht [sic!] auf der Stimmabgabe der einzelnen Mitglieder ... für oder gegen einen bestimmten Beschlussantrag. ... Der Beschluss beruht also auf zumindest einer Willenserklärung und führt eine Rechtsfolge herbei, so dass er als (mehrseitiges) Rechtsgeschäft einzuordnen ist.“

12 O. Fn. 7, S. 177 f. u. öfter.

13 H. Dröge, Haftung für Gremienentscheidungen, 2008, S. 18 ff.

14 W. Flume, Die juristische Person, 1983, S. 249. Dagegen hatte Helmut Coing schon 1957 angemerkt, der Beschluss sei ein einseitiges Rechtsgeschäft, wobei freilich Coing weiter davon ausging, der Beschluss setze sich aus den Stimmabgaben zusammen; Coing, in: Staudinger, BGB, Bd. I - Allgemeiner Teil, 11. Aufl., 1957, \32 Rn. 28. Die Ansicht Coings hat indes die weitere Diskussion des Beschlussbegriffs nicht beeinflusst.

15 O. Fn. 6 a.a.O. 
sonen" verweist hingegen die aktuelle Darstellung des Allgemeinen Teils von Detlef Leenen: „Der Beschluss ist ein mehrseitiges Rechtsgeschäft, das durch die gleichgerichteten Willenserklärungen der an der Beschlussfassung beteiligten Personen zustande kommt." ${ }^{16}$ Was sich aus dem herkömmlichen Beschlussverständnis für die Beschlussfeststellung ergibt, dass diese nämlich für die Rechtswirkung des Beschlusses im Grundsatz unbeachtlich ist, hat vor einiger Zeit Wolfgang Zöllner erneut ausgeführt. ${ }^{17}$

Die folgenden Ausführungen zielen auf eine kritische Überprüfung des Ansatzes, der den Beschluss als ein mehrseitiges Rechtsgeschäft begreift, das durch die Gesamtheit der einzelnen Stimmabgaben konstituiert oder bewirkt wird. Zur Literaturlage siehe noch das P.S. Seite 42.

\section{Die Beschlussfassung im und für das Verbandsorgan und Mehrheitsentscheidungen ohne Organbezug und -grundlage}

Es besteht ein wesentlicher Unterschied zwischen der Beschlussfassung, die im und für das Verbandsorgan erfolgt, und solchen Mehrheitsentscheidungen, bei denen die Abstimmenden nicht aufgrund einer Organzugehörigkeit tätig werden. ${ }^{18}$ Ein Musterbeispiel für die Abstimmung, bei der die Stimmberechtigten nicht für ein Verbandsorgan handeln, ist die Abstimmung unter Bruchteilseigentümern nach $\mathbb{S} \mathbb{S} 741 \mathrm{ff}$. BGB. ${ }^{19}$ Für die Abstimmung unter Bruchteilseigentümern dürfte der hier auf von Tubr zurückgeführte Beschlussbegriff im Wesentlichen zutreffen: Wenn die Mehrheit der Bruchteilseigentümer eine Verwaltungsmaßnahme beschließt (\$ 745 I BGB), dann müssen die überstimmten Miteigentümer die Vornahme der entsprechenden Verwaltungsmaßnahme gegen sich gelten lassen; sie sind nicht kraft der Mehrheitsentscheidung notwendig selbst an der Durchführung der Verwaltungsmaßnahme beteiligt, die Durchführung ist vielmehr eine Angelegenheit nur derjenigen, die mit JA gestimmt haben. ${ }^{20}$ Die überstimmten Miteigentümer sind nicht ihrerseits Mit-Urheber der Mehrheitsentscheidung, diese nimmt ihnen lediglich das Recht, sich der Durchführung der Maßnahme zu

${ }^{16}$ D. Leenen, BGB Allgemeiner Teil: Rechtsgeschäftslehre, 2011, S. 32.

17 O. Fn. 7; vor Zöllner s. bereits Baltzer (o. Fn. 7), S. 175 f.: Beschlussfeststellung „lediglich ein zu der verfahrensmässig bereits existenten Größe ,Beschluss` hinzutretender Akt der Ausführung [sic!] des Beschlusses“.

${ }^{18}$ Grundlegend zur Organschaft im Recht der privaten Verbände J. Schürnbrand, Organschaft im Recht der privaten Verbände, 2007; s. auch Jacoby (o. Fn. 11).

19 S. dazu umf. R. Schnorr, Die Gemeinschaft nach Bruchteilen ( $\$ \$ \$ 741-758$ BGB), 2004, S. 238 ff.; seither vor allem Jacoby (o. Fn. 11), S. $111 \mathrm{ff}$.

${ }^{20}$ W. Flume, Die Personengesellschaft, 1977, S. 116 f.; Schnorr (o. Fn. 19), S. 241, dort auch Auseinandersetzung mit abw. Ansichten. 
widersetzen. ${ }^{21}$ Insbesondere bei der Ausführung des Beschlusses unterscheidet sich hier in der Tat die Rechtsstellung derjenigen, die die Mehrheit ausmachen, von der Rechtsstellung derjenigen, die sich als Minderheit nicht haben durchsetzen können. Mehrheitsentscheidungen, die nicht für ein Organ gefasst werden, werden im Gesetz üblicherweise nicht als Beschluss bezeichnet. ${ }^{22}$ Vielmehr spricht z.B. $\$ 745$ I BGB von der „Stimmenmehrheit“. Die „Stimmenmehrheit“ ist nicht ein Beschluss der Gemeinschaft nach Bruchteilen. Es dürfte sich empfehlen, Mehrheitsentscheidungen wie die nach $\$ 745$ I BGB nicht als Beschluss zu bezeichnen. Beschluss ist demnach nur diejenige Mehrheitsentscheidung, die für ein Verbandsorgan getroffen wird und aus einer Aktivität dieses Verbandsorgans hervorgeht. Die Mehrheitsentscheidung, die nicht für ein Organ und von diesem getroffen wird, aber auch nur diese - wie man sagen könnte: - „schlichte“ Mehrheitsentscheidung, lässt sich als das zusammengesetzte, mehrseitige Rechtsgeschäft erfassen, das von Tuhr mit dem Beschluss identifiziert hat.

Die These des folgenden Beitrags geht dahin, dass der Beschlussbegriff, welcher den Beschluss mit der Gesamtheit der übereinstimmend abgegebenen JA-Stimmen identifiziert, für die Erfassung der Beschlüsse von Verbandsorganen nicht geeignet ist. Im Folgenden ist daher nur noch von der Beschlussfassung die Rede, die im Rahmen der Tätigkeit eines Verbandsorgans und für dieses erfolgt. Die schlichte Mehrheitsentscheidung, die m.E. auch kein Beschluss ist, ist nicht Gegenstand dieses Beitrags. Abkürzend wird hier von der "Versammlung“ gesprochen, doch sind auch Organe wie der mehrgliedrige Vorstand, der Aufsichtsrat, ein Stiftungsrat und dergleichen gemeint. Es wäre zu überlegen, inwieweit die vorgestellten Thesen auch für die Beschlussfassung unter Gesellschaftern einer Personengesellschaft gelten können, soweit es um die Herstellung eines gemeinsamen Willens für die „Wirkungseinheit“ geht und nicht allein um das Verhältnis der Gesellschafter zueinander. ${ }^{23}$ Es kommt darauf an, ob man die Versammlung der BGB-Gesellschafter als Organ der Gesellschaft begreifen kann; diese Frage wird hier nicht untersucht. Wenn für die folgenden Überlegungen der Anspruch erhoben wird, sie seien rechtsformunabhängig gültig, versteht sich dies hinsichtlich der verschiedenen Erscheinungsformen solcher Verbände des Privatrechts, bei denen von Organen die Rede sein kann. Es geht mithin

${ }^{21}$ Zutr. G.-H. Langhein, in: Staudinger, BGB (2008), \$ 745 Rn. 28.

${ }^{22}$ Zutr. hat sich Medicus dagegen gewandt, das „Einvernehmen“ der sorgeberechtigten Eltern nach $₫ 1627$ S. 1 BGB als „Beschluss“ zu bezeichnen; s.o. Fn. 6 a.a.O.

${ }^{23} \mathrm{Zu}$ dieser Unterscheidung s. Flume (o. Fn. 20), S. 97 ff.; zur Beschlussfassung in der Personengesellschaft s. auch U. Noack, Fehlerhafte Beschlüsse in Gesellschaften und Vereinen, 1989, 169 ff.; D. Leenen, Festschrift Larenz, 1983, S. 371 ff.; zum Handeln der Gesellschafter der Personengesellschaft nach Art eine „Organs“ s. auch Schürnbrand (o. Fn. 18), S. $90 \mathrm{ff}$. 
um die Beschlussfassung in Versammlungen der Mitglieder/Gesellschafter/ Genossen/Wohnungseigentümer, aber auch um die Beschlussfassung in Vorstand und Aufsichtsrat, im Betriebsrat, in Gläubigerausschuss wie -versammlung nach der Ins $\mathrm{O}$ und dergleichen.

Im Bereich des Privatrechts hat man die Lehre vom Beschluss in die Lehre vom Rechtsgeschäft einzuordnen, unabhängig davon, ob man im Beschluss ein Rechtsgeschäft stricto sensu sehen mag. Im Folgenden geht es darum, den Beschluss, wie er in organisierten Verbänden vorkommt, als Handlungsform mit der zivilistischen Lehre vom Rechtsgeschäft zu koordinieren. ${ }^{24}$

\section{Positiv- und Negativbeschlüsse}

Ist die Ablehnung eines Antrags auch ein Beschluss? Ausgehend von dem Standpunkt, der den Beschluss mit den ihn tragenden Stimmen identifiziert, muss dies fraglich erscheinen. Sehen wir zunächst auf eine vergleichbare Situation beim Vertragsschluss: Wird ein Angebot zum Abschluss eines Vertrages nicht angenommen, sondern abgelehnt, so ist ein Vertrag nicht zustande gekommen. Nur insofern hat auch die Ablehnung eines Angebots eine gewisse Rechtswirkung, als sie das u.U. noch annahmefähige Angebot beseitigt. ${ }^{25}$ Der Nicht-Vertrag enthält aber keine Regelung. Beim Beschluss verhält es sich anders: $Z$ war enthält nur der Positivbeschluss, durch den der zur Abstimmung gestellte Antrag angenommen wird, eine für den Verband getroffene Festlegung für das weitere Verbandsgeschehen, gleichgültig, ob es noch weiterer Umsetzungsaktivitäten bedarf oder nicht. Aber auch der Negativbeschluss gilt zu Recht als ein Beschluss, weil durch ihn für den Verband die Festlegung getroffen wird, dass der Antrag nicht gewollt ist. ${ }^{26}$ Mit Recht gilt im Aktienrecht der negative Beschluss als möglicher Anfechtungsgegenstand. ${ }^{27}$ Ebenso verhält es sich nach der Erkenntnis des Bundesgerichtshofs auch bei Negativbeschlüssen der Wohnungseigentümerversammlung; diese seien keineswegs blosse Nichtbeschlüsse. ${ }^{28}$ Für uns ist nun bedeutsam, dass ein Negativbeschluss nicht als ein „Zusammenwirken mehrerer inhaltlich übereinstimmender Willenserklärungen, also [als] ein mehrseitiges Rechtsgeschäft“ erfasst werden kann: Wenn ein Antrag „durchgefallen“ ist, weil

${ }^{24}$ Vielleicht sind die anschließend zu entwickelnden Thesen für die Beschlusslehre doch auch auf dem Gebiet des Öffentlichen Rechts anschlussfähig.

$25 \$ 146,1$. Alt. BGB.

26 Siehe Zöllner (o. Fn. 7), S. 821, 823; Dröge (o. Fn. 13), S. 22 m.w.N.; a.A. R. Winnefeld, DB 1972, 1053 ff., bei dem überhaupt die von Tuhr'sche Beschlusslehre in ihren Konsequenzen eindrucksvoll durchbuchstabiert ist.

27 BGHZ 97, 28.

${ }^{28}$ BGHZ 148, 335, $341 \mathrm{ff}$. 
keine hinreichende Anzahl von JA-Stimmen abgegeben wurden, kann dem auf der „Ablehnungsseite“ ein mixtum compositum aus NEIN-Stimmen, Enthaltungen und ungültigen Stimmen gegenüberstehen: Die Gesamtheit der NEIN-Stimmen, Enthaltungen und ungültigen Stimmen, von der man sagen kann, sie habe die Annahme des Antrags verhindert, ${ }^{29}$ ist gerade keine Vereinigung inhaltlich übereinstimmender (gleichlautender) Willenserklärungen. Besonders deutlich wird dies, wenn es zum Negativbeschluss kommt, weil ein erforderliches „Mitgliedermehr“ nicht erreicht wurde: ${ }^{30}$ Verfehlt etwa der Antrag auf Entziehung des Wohnungseigentums das erforderliche, überhälftige Mehr der stimmberechtigten Wohnungseigentümer ( $(18$ WEG), dann sind u.U. für den Negativbeschluss neben den NEIN-Stimmen und den Enthaltungen auch diejenigen Wohnungseigentümer ursächlich geworden, die nicht zur Versammlung erschienen sind. Das Fernbleiben von der Versammlung ist aber als solches kein rechtsgeschäftliches Handeln und kann daher nicht „Bestandteil“ oder „Entstehungselement“ des ablehnenden Beschlusses sein. Die Anerkennung des Negativbeschlusses als Beschluss ist mit der von Tuhr'schen Beschlusslehre nicht zu vereinbaren. Mit der Anerkennung des Negativbeschlusses als Beschluss ist in Wahrheit der von Tubr'sche Beschlussbegriff bereits aufgegeben worden.

\section{Der Beschluss als Rechtsakt des Verbandsorgans}

Da nach der hier vertretenen Auffassung der Beschluss als Rechtsakt nicht schon mit der Gesamtheit der einzelnen (zustimmenden) Stimmabgaben gegeben ist, muss gefragt werden, von wem der Beschluss ausgeht, wer dessen auctor ist. Denn wenn der Beschluss nicht aus der Gesamtheit der ihn tragenden Stimmabgaben zusammengesetzt ist, kann man für die Urheberschaft des Beschlusses nicht einfach auf die zustimmenden Mitglieder verweisen.

Der Beschluss ist ein Rechtsakt der Versammlung als ganzer, nicht nur der Abstimmungsmehrheit derjenigen, die für den Beschluss gestimmt haben. Der Beschluss ist Ergebnis eines Handelns des Organs „Versammlung“,

${ }^{29} \mathrm{Ob}$ Enthaltungen der Annahme eines Antrags entgegenwirken, hängt vom dem konkreten Mehrheitserfordernis ab: Gilt das Anwesendenmehr (vulgo: absolutes Mehr), wie es nach der gesetzlichen Regelung für den Verein bestimmt schien ( $\$ 32$ I 3 BGB), wirken Enthaltungen der Annahme des Antrags entgegen; stellt man auf das Überwiegen der JA-Stimmen über die NEIN-Stimmen ab (so BGHZ 83, 35 für $\ 32$ I 3 BGB; BGHZ 106, 179 für $\$ 25$ WEG), entfalten nur die NEIN-Stimmen eine Gegenwirkung, nicht aber die Enthaltungen.

${ }^{30}$ Ebenso kann auf die Fälle verwiesen werden, in denen ein besonderes Beschlussfassungsquorum erforderlich ist. 
nicht nur der den Beschluss befürwortenden Mehrheit. ${ }^{31}$ Die Versammlung, die als Urheber des Beschlusses anzusehen ist, ist wohlgemerkt nicht die Gruppe der bei der konkreten Abstimmung anwesenden und mitstimmenden Mitglieder, sondern das Verbandsorgan „Versammlung“, als das die Anwesenden, falls beschlussfähig, agieren. Die in der konkreten Versammlung Agierenden sind im Verhältnis zum Organ die Organwalter. ${ }^{32}$ Die Zurechnung des Beschlusses zur Versammlung ist auch die gedankliche Voraussetzung dafür, dass der Verein nach $\$ 31$ BGB wegen eines Beschlusses der Mitgliederversammlung haftbar gemacht werden kann, wenn die Beschlussfassung eine Handlung darstellt, die einen Dritten zum Schadensersatz berechtigt. ${ }^{33}$

Dass die Versammlung der Urheber des Beschlusses ist, zeigt sich schön an dem Vorgang der Aufhebung eines Beschlusses, also am sog. Konträrakt. Hierzu genügt, ist aber auch erforderlich, dass wieder mit der nötigen Mehrheit entschieden wird; es ist nicht erforderlich, dass genau diejenigen Mitglieder für die Beschlussaufhebung stimmen, deren Stimmen für die ursprüngliche Fassung des Beschlusses gesorgt haben. Wenn also in einer Versammlung, in der die fünf Mitglieder A, B, C, D und E anwesend sind, A, B und C für einen Antrag gestimmt haben, so wäre der Beschluss nach der durch von Tubr formulierten Ansicht ein Rechtsgeschäft nur von A, B und C, weil nur diese gleichlautende, den Beschluss befürwortende Stimmen abgegeben haben. Nach der hier vertretenen Ansicht ist der Beschluss ein Rechtsakt der Versammlung als ganzer; für die Rechtswirkung des Beschlusses kommt es grundsätzlich nicht mehr darauf an, welche konkreten Mitglieder für ihn gestimmt haben. ${ }^{34}$ Angenommen, bei der späteren Behandlung eines Aufhebungsantrags sind noch zahlreiche weitere Mitglieder anwesend; A, B und C befürworten die Aufhebung „ihres“ Beschlusses, bleiben damit aber in der Minderheit: Hier unterbleibt die Beschlussaufhebung. Umgekehrt kann eine Mehrheit den Beschluss jetzt aufheben, auch wenn dabei A, B und C gegen die Beschlussaufhebung stimmen. Wäre der Beschluss ein Rechtsgeschäft derjenigen, die ihn durch ihre JA-Stimme verabschiedet haben, müsste bei diesen auch die Zuständigkeit für die Aufhebung liegen.

${ }^{31}$ Zutr. Dröge (o. Fn. 13), S. 20 f. - Die aktienrechtliche Anfechtungsklage richtet sich nicht gegen diejenigen, die dem anzufechtenden Beschluss zugestimmt haben, sondern gegen die Aktiengesellschaft, deren Organ Hauptversammlung den anzufechtenden Beschluss mit Wirkung für die Gesellschaft gefasst hat; $\$ 246$ II 1 AktG.

32 Grundlegend Schürnbrand (o. Fn. 18), S. 231 ff.; Jacoby (o. Fn. 11), S. 163 f.; s. auch V. Beuthien, Festschrift Zöllner, Bd. 1, 1998, S. 87 ff.

${ }^{33}$ Zur Außenhaftung des Vereins für Akte der Mitgliederversammlung s. (krit.) Renter, in: MünchKomm-BGB, 6. Aufl. 2012, $\$ 31$ Rn. 24 m.w.N.; Schürnbrand (o. Fn. 18), S. 99 ff.

${ }^{34}$ Es verhält sich auch nicht so, dass diejenigen, die gleichgerichtete Stimmerklärungen abgeben, unter sich irgendein Rechtsverhältnis begründen würden. 
Es ist immer wieder zu lesen, dass der Beschluss mit der Abgabe der letzten an der Abstimmung beteiligten Stimme zustande komme. ${ }^{35}$ Diese Sichtweise passt zu der Ansicht, der Beschluss sei die Vereinigung mehrerer inhaltlich übereinstimmender Willenserklärungen. ${ }^{36}$ Nach der hier vertretenen Ansicht kann mit der Abgabe der letzten an der Abstimmung beteiligten Stimme der Beschluss noch nicht gefasst sein. Es fehlt zu diesem Zeitpunkt noch an dem unverzichtbaren Endakt, durch den die Stimmabgaben in eine Entscheidung umgesetzt werden, die als Entscheidung der Versammlung als ganzer gelten kann. ${ }^{37}$ Das Erfordernis der Beschlussfeststellung ergibt sich schon daraus, dass der Beschluss ein Rechtsakt der Versammlung als ganzer ist, während die Stimmabgaben ein Rechtshandeln der einzelnen Mitglieder darstellen. Nach Abgabe der Stimmen steht allerdings schon fest, mit welchem inhaltlichen Ergebnis der Beschluss - korrektes Vorgehen vorausgesetzt - festzustellen sein wird. Auch wenn mit dem Abschluss der Abstimmung die Grundlage der anschließenden Beschlussfeststellung fixiert ist, ist der Beschluss doch erst gefasst mit und durch seine Feststellung.

Die Unverzichtbarkeit der Beschlussfeststellung wird durch eine funktionale Betrachtung des Beschlusses unterstützt: Mit dem Beschluss wird ein für den Verband bindender Willensentschluss gefasst, der dem Verband - bildlich gesprochen - eine Marschrichtung vorgibt. Dadurch wird das auf den Verband bezogene Verhalten der anderen Organe und der Mitglieder angeleitet. Der Beschluss hat Steuerungsfunktion. Nehmen wir an, vier Gesellschafter haben ihre Stimmen abgegeben, wobei aber Streit besteht, ob ein Stimmrechtsausschluss eine oder mehrere dieser Stimmabgaben ungültig macht. Man kommt zu keiner Einigung und geht auseinander. Mit dem Beschlussverständnis, das den Beschluss mit der Gesamtheit der zustimmenden Stimmabgaben gleichsetzt, müsste man nun allerdings sagen, der Beschluss sei gefasst ungeachtet dessen, dass über den Beschlussinhalt (Annahme oder Ablehnung des Antrags) keine Einigkeit besteht. Ein solcher „Beschluss“ kann aber die Funktion, das Handeln der Verbandsorgane und -mitglieder zu steuern, nicht erfüllen. Ähnlich verhält es sich, wenn man die Versammlung beendet, ohne festgestellt zu haben, welcher Beschlussinhalt aus der Gesamtheit der Stimmabgaben resultiert. Wenn man zum Beispiel auseinandergeht und die Urne mit den eingesammelten Stimmzetteln für eine spä-

${ }^{35}$ Z.B. Roth, in: Roth/Altmeppen, GmbHG, 6. Aufl. 2009, $\$ 47$ Rn. 4.

${ }^{36}$ Bei sukzessiver Stimmabgabe müsste danach, genau besehen, der Positivbeschluss sogar schon mit der letzten zustimmenden Stimmabgabe zustande gekommen sein (oder sogar schon mit Abgabe der Stimme, mit der das Mehrheitserfordernis erfüllt wird); der Negativbeschluss mit derjenigen Stimmabgabe, nach der feststeht, dass die erforderliche Mehrheit mit den ausstehenden Stimmen keinesfalls mehr erreicht werden kann.

37 Für die GmbH ist dies nicht die h.M.; vgl. Hüffer, in: GroßKomm-GmbHG, 2006, \47 Rn. 26 m. umf. Nachw. 
tere Auszählung wegschließt, so müsste nach dem von Tubr'schen Beschlussbegriff ein Beschluss bereits gefasst sein. Die Lage, wie sie hier besteht, gibt aber den Verbandsorganen und -mitgliedern nicht die mindeste Anleitung, was zu tun und was zu unterlassen ist. Ein Beschlussbegriff, der die Steuerungsfunktion des Beschlusses im Verband ignoriert und auch in Situationen, in denen von einer den Verband anleitenden Willensbildung noch keine Rede sein kann, das Gegebensein eines Beschlusses bejaht, empfiehlt sich nicht.

Es verhält sich auch keineswegs so, dass jeder, der einer Stimmabgabe gefolgt ist, nun auch genau wissen müsste, was das inhaltliche Beschlussergebnis ist: Immer bedarf es einer - größeren oder kleineren - gedanklichen juristischen Leistung, die von dem realweltlichen, äußerlich wahrnehmbaren Heben einer gewissen Anzahl von Händen, Stimmkarten oder dergleichen zu der Feststellung führt, der Antrag sei von der Mehrheit angenommen oder abgelehnt. Diesen Schritt leistet erst die Beschlussfeststellung, die aus den nackten Zahlen des Abstimmungsergebnisses den Inhalt des Beschlusses herleitet. Es ist einzuräumen, dass der in der Beschlussfassung eingeschlossene Vorgang der Rechtsanwendung auch ganz einfach, geradezu trivial sein kann; dies ändert aber nichts daran, dass ein juristischer Gedankenschritt stets notwendig ist. ${ }^{38}$ Diese Notwendigkeit, von den in der Abstimmung ermittelten Zahlen für „JA“, „NEIN“, „Enthaltung“ und „ungültig“ gedanklich zum Beschlussinhalt zu kommen, besteht bei jeder Beschlussfassung, erfolge sie nun in der Vereinsversammlung oder in der Hauptversammlung der Aktiengesellschaft. Zwischen den mittels Stimmabfrage erhobenen Zahlen und dem Beschluss liegt immer und notwendig ein Hiatus, nicht nur bei den Versammlungen der Aktionäre, sondern in allen mehrgliedrigen Organen, die einen Willen im Abstimmungswege bilden.

Beim Vertragsschluss durch Angebot und Annahme sagen wir allerdings nicht, dass erst ein zusätzlicher Akt einer gemeinschaftlichen „Konsensfeststellung“ den Vertrag zustande bringt. Wir überlassen es vielmehr den Vertragsschließenden, je für sich und unabhängig voneinander zu beurteilen, ob durch fristgerechten Zugang einer uneingeschränkten Annahmeerklärung ein ordnungsgemäß gemachtes Angebot angenommen wurde und dadurch der Vertrag zustande gekommen ist. ${ }^{39}$ Wenngleich sich erst durch eine Rechtsanwendung der gültige Abschluss des Vertrages erkennen lässt, bewirken nach unserer Vorstellung Angebot und Annahme den Vertrag doch ohne einen zusätzlichen gemeinschaftlichen Akt der „Konsensfeststellung“. Ungeachtet dessen, ob der einzelne Kontrahent die Rechtslage zutreffend einschätzt, steht objektiv fest, ob eine Vertragsbindung gegeben ist oder nicht.

38 Zutr. Zöllner (o. Fn. 7), S. 825 f.

${ }^{39}$ Das Gesetz sorgt nur dafür, dass den Vertragsschließenden hierfür die erforderlichen Informationen zustehen, s. $\$ 149$ S. 1 BGB. 
Wer als Kontrahent einen gültigen Vertrag als ungültig einschätzt, wird daher in Gläubigerverzug und - wegen der grundsätzlichen Unbeachtlichkeit des Rechtsirrtums - in Schuldnerverzug kommen. Wer als Kontrahent einen ungültigen Vertrag als gültig einschätzt, wird möglicherweise eine Leistung ohne Rechtsgrund erbringen und den anderen Teil ungerechtfertigt zur Leistung auffordern. Für die Beschlussfassung kann man es jedoch nicht so halten wie beim Vertragsschluss: Während es beim Vertragsschluss nur um die beiden Parteien geht, die den Vertragsschluss betreiben und daher auch dessen Stand beurteilen können, bindet der gefasste Beschluss weitere Rechtssubjekte, die nicht an der Beschlussfassung beteiligt waren, nämlich die abwesenden Mitglieder und die anderen Organe des Verbandes. Den - ebenfalls gebundenen - abwesenden Mitgliedern und auch den anderen Verbandsorganen ist eine eigenständige Beurteilung, mit welchem Ergebnis in der Versammlung Stimmen abgegeben wurden, nicht möglich. Schon deswegen liegt es bei der Beschlussfassung anders als beim Vertragsschluss, verlangt die Beschlussfassung nach einer überpartikularen Feststellung des Beschlussergebnisses, an die sich alle vom Beschluss Betroffenen halten können. Die Beschlussfeststellung ist damit nicht nur die nachfolgende Verkündung eines Rechtsgeschäfts, das als solches bereits abgeschlossen und auch ohne Verkündung existent ist, sondern es perfiziert die Beschlussfeststellung überhaupt erst den Beschluss. ${ }^{40}$ Dabei handelt es sich auch nicht bloß um ein äußeres Wirksamkeitserfordernis, das erfüllt werden muss, um einem inhaltlich schon feststehenden Rechtsgeschäft Wirksamkeit zu verleihen. Die Beschlussfeststellung entscheidet nicht nur über das „Ob“, sondern auch über den Inhalt des Rechtsakts „Beschluss“ und bildet daher ein echtes Tatbestandsmerkmal der Beschlussfassung.

Wenn man anerkennt, dass der Beschluss auf der Beschlussfeststellung als einem konstitutiven Endakt des Verfahrens der Beschlussfassung beruht, ist auch der Negativbeschluss zwanglos als Beschluss zu erfassen. Ebenso stellt es nach der hier vertretenen Ansicht keine Schwierigkeit dar, dass auch der durch einen Alleingesellschafter oder durch das einzige erschienene Mitglied (Beschlussfähigkeit vorausgesetzt) gefasste Beschluss ein gewöhnlicher Beschluss ist, der als etwaiger Anfechtungsgegenstand usf. nicht anders behandelt wird als ein Beschluss, der von einer Mehrzahl von Stimmberechtigten erzeugt worden ist: So oder so handelt es sich immer um einen Rechtsakt der Versammlung als Verbandsorgan.

Für die Hauptversammlung der Aktiengesellschaft ist in ständiger Praxis anerkannt, dass bei unterbliebener Beschlussfeststellung nicht die Anfechtungsklage - die eben einen Beschluss als Streitgegenstand voraussetzt -, sondern die allgemeine Feststellungsklage anzustellen ist. ${ }^{41}$ Nach der Rechtspre-

${ }^{40}$ Grds. a.A. Zöllner (o. Fn. 7), S. 826 f.

${ }^{41}$ BGHZ 76, 154, 156; BGH NJW 1996, 259; s. aber noch unten XIII. 
chung des Bundesgerichtshofs soll sich indes die Fassung des Beschlusses in der Vereinsversammlung und in der Hauptversammlung der Aktiengesellschaft kategorial unterscheiden. Für das Aktienrecht verhalte es sich so, dass das von zuständiger Stelle, typischerweise also durch den Versammlungsleiter, festgestellte Beschlussergebnis den maßgeblichen Beschlussinhalt angebe. ${ }^{42}$ Dabei verhält es sich nicht bloß so, dass der festgestellte Beschluss aus Gründen des Vertrauensschutzes und dergleichen als vorläufiger Rechtsschein des „wirklichen“ Beschlusses zu nehmen wäre: Der Beschluss selbst ist erst mit und aufgrund der Beschlussfeststellung - und also mit dem ihm dabei gegebenen Inhalt - gefasst.

Freilich soll die Beschlussfeststellung nur für die Aktiengesellschaft entscheidend sein, nicht hingegen für den Verein. ${ }^{43}$ Zur Begründung wird darauf verwiesen, dass bei der Aktiengesellschaft - im Gegensatz zum Verein - die Beschlussfassung zu einem konstitutiven, das Ergebnis fixierenden Akt führe; dies wiederum ergebe sich im Aktienrecht daraus, dass die Feststellung des Beschlussergebnisses vor allem Bedeutung für die Geltendmachung von Mängeln habe. Wegen der Bindung der Anfechtungsklage an die kurze Frist von einem Monat ( $\$ 246$ I AktG), müssten die Anfechtungsberechtigten von einem bestimmten Beschlussergebnis als maßgebend ausgehen können; dazu sei erforderlich, dass dieses Ergebnis festgestellt und verkündet wird. Entgegen dieser Rechtsprechung ist die Notwendigkeit einer Beschlussfeststellung nicht eine Besonderheit der Hauptversammlung der Aktiengesellschaft, sondern ein für jeden Beschlussvorgang von der Sache her erforderlicher Schritt. $^{44}$

Auf die vereinsrechtliche Frage der entsprechenden Anwendung der $\mathbb{S} 241 \mathrm{ff}$. AktG soll hier nicht eingegangen werden. ${ }^{45}$ Unabhängig davon, ob man die $\mathbb{\int} 241 \mathrm{ff}$. AktG auf die Beschlüsse der Vereinsversammlung anwendet, ist doch der Hergang der Beschlussfassung durch die Vereins- bzw. die Hauptversammlung nicht als kategorial gegensätzlich zu erfassen. ${ }^{46}$ Das Erfordernis einer Beschlussfeststellung - im Sinne einer gedanklichen Ableitung des Beschlussinhalts aus dem numerischen Abstimmungsergebnis besteht für diese Versammlungen unterschiedslos. Man sollte daher auch für

${ }^{42}$ BGHZ 104, 66; die ältere, zu Recht aufgegebene Rechtsprechung wollte diese Wirkung noch davon abhängig machen, dass der Beschluss beurkundet worden ist: BGHZ 51, 209, $211 \mathrm{ff}$.

43 BGH NJW 1975, 2101.

${ }^{44}$ Für die Beschlüsse der Eigentümerversammlung nach WEG begründet BGHZ 148, 335, 343 ff. den konstitutiven Charakter der Beschlussfeststellung ebenfalls mit der gesetzlichen Befristung der Beschlussanfechtung.

${ }^{45}$ Ausf. Reuter, in: MünchKomm-BGB, 6. Aufl. 2012, $\$ 32$ Rn. 55 ff. m. umf. Nachw.

${ }^{46}$ Sollte sich wirklich die Rechtsnatur der Beschlussfassung der Vereinsversammlung kategorial ändern, wenn der Gesetzgeber für Klagen gegen die Beschlüsse der Vereinsversammlung eine Frist einführen würde? 
den Verein davon ausgehen, dass eine Beschlussfassung zwingend die Feststellung des Beschlussergebnisses erfordert. Die weitere Frage, ob die Beschränkung von Angriffen auf Vereinsbeschlüsse mittels einer Analogie zu $\mathbb{S} 241 \mathrm{ff}$. AktG bewerkstelligt wird oder auf andere Weise, soll damit nicht präjudiziert sein.

\section{Die Zuständigkeit für die Beschlussfeststellung}

$\mathrm{Zu}$ fragen ist nun nach der Zuständigkeit für die Beschlussfeststellung. Sehen wir zunächst auf die Versammlung, deren Verfahren von einem Versammlungsleiter geordnet wird. Die Feststellung des Beschlusses ist - wie die Durchführung der vorausgegangenen Abstimmung - ein Ordnungshandeln, für das grundsätzlich der Versammlungsleiter zuständig ist. Der Beschluss ist daher vom Versammlungsleiter festzustellen. Der Beschluss ist freilich kein Rechtsakt des Versammlungsleiters; dieser eröffnet den Beschluss vielmehr für die Versammlung als ganze: Die Beschlussfeststellung wird, auch wenn sie vom Versammlungsleiter vorgenommen wird, als Rechtsakt der Versammlung als ganzer - und über diese dem Verband - zugerechnet. Die Feststellung des Beschlusses durch den Versammlungsleiter ist ein gebundenes Rechtshandeln: Das Abstimmungsergebnis, so wie es vom Versammlungsleiter erhoben worden ist, und damit die Gesamtheit der einzelnen Stimmabgaben, determiniert im Zusammenspiel mit gesetzlichen und satzungsmäßigen Regelungen vor allem über die Beschlussfähigkeit, das Mehrheitserfordernis, über die Stimmgültigkeit und ein etwaiges Quorum, mit welchem Inhalt der Beschluss festzustellen ist. Hierzu ist geradezu selbstverständlich nicht erforderlich, dass der Versammlungsleiter für seine Feststellung die Zustimmung aller an der Abstimmung teilnehmenden Mitglieder erhält, wie Zöllner es für die $\mathrm{GmbH}$ verlangt hat. ${ }^{47}$

Die von einem Versammlungsleiter durchgeführte Versammlung verliert allerdings nicht die Verfahrenshoheit über ihr eigenes Vorgehen. ${ }^{48}$ Soweit nicht die Rechtsgrundlagen entgegenstehen, erscheint es daher denkbar, dass die Versammlung mittels eines Ordnungsantrags das Geschäft der Beschlussfeststellung an sich zieht. In diesem Fall entscheidet anschließend die Versammlung als ganze, wie das Beschlussergebnis inhaltlich zu lauten hat. Bei einer Versammlung, die ohne Versammlungsleiter durchgeführt wird, muss der Beschluss ohnehin durch die versammelten Mitglieder festgestellt wer-

47 Zöllner (o. Fn. 7), S. 827 f.; ebenso ders., GmbHG, 19. Aufl. 2010, 47 Anh. Rn. 120.

${ }^{48}$ Zum Verhältnis von Ordnungsgewalt des Versammlungsleiters und Verfahrenshoheit der Versammlung s. W. Ernst, Kleine Abstimmungsfibel, 2011, Rn. 10; Schürnbrand (o. Fn. 18), S. $136 \mathrm{ff}$. 
den. Wenn bei Fehlen eines Versammlungsleiters oder nach Devolution der Beschlussfeststellung vom Versammlungsleiter auf die Versammlung als ganze Meinungsverschiedenheiten darüber auftreten, mit welchem Inhalt der Beschluss festzustellen ist, muss durch Abstimmung entschieden werden. Für diesen Fall ist umstritten, ob die Beschlussfeststellung Einstimmigkeit erfordert ${ }^{49}$ oder ein Mehrheitsbeschluss genügt. ${ }^{50}$ Es handelt sich um eine Ordnungsentscheidung und Ordnungsentscheidungen werden - wo nichts Abweichendes bestimmt ist - mit einfacher Mehrheit getroffen. Für das Verlangen nach Einstimmigkeit müsste eine gesetzliche oder satzungsmäßige Grundlage angegeben werden. Derlei ist nicht bekannt. In Wahrheit wäre das Mehrheitsprinzip aufgegeben, wenn man für die Beschlussfeststellung Einstimmigkeit fordern würde. Gegenstand der zu treffenden Ordnungsentscheidung ist die Feststellung, zu welchem Beschlussinhalt die vorausgegangene Sachabstimmung geführt hat. In diesen Fällen, in denen der Versammlung als ganzer die Beschlussfeststellung obliegt, handelt es sich auch für die Versammlung als ganze - ebenso wie sonst für den Versammlungsleiter - um ein gebundenes Rechtshandeln: Auch die den Beschluss feststellende Versammlung hat pflichtgemäß denjenigen Beschlussinhalt zu bestimmen, der sich rechtlich aufgrund des Vergleichs der gezählten und summierten JA- und NEIN-Stimmen sowie der Enthaltungen und der ungültigen Stimmen mit den Quorums- und Mehrheitserfordernissen ergibt. Die Mitglieder der Versammlung dürfen hierbei - anders als bei der vorausgegangenen Sachabstimmung - nicht willkürlich vorgehen. Eine Beschlussfeststellung, die das Ergebnis der zugrunde liegenden Sachabstimmung ignorieren oder offensichtlich verfälschen würde, wäre als nichtig anzusehen.

Auch wenn die Versammlung als ganze den Beschluss feststellt, muss dies mit einer gewissen Förmlichkeit geschehen ${ }^{51}$ es bedarf einer ausdrücklichen Feststellung. Eine konkludente Beschlussfeststellung ist nicht denkbar, weil ein Beschlussinhalt zu fixieren ist, den man den abwesenden Mitgliedern oder anderen Verbandsorganen mitteilen kann..$^{52}$ Die Vorstellung, man könne sich auf einen Beschluss berufen, dessen Existenz einfach darauf beruhen soll, dass man sich irgendwie schon einig gewesen sei, ist absurd. ${ }^{53}$ Beschluss-

${ }^{49}$ Hierfür (betr. Gesellschafterbeschlüsse der $\mathrm{GmbH}$ ): Zöllner, in: Baumbach/Hueck, GmbHG, 19. Aufl. 2010, 47 Anh. Rn. 120 m.w.N.

${ }^{50}$ Hierfür (betr. Gesellschafterbeschlüsse der $\mathrm{GmbH}$ ): Roth, in: Roth/Altmeppen, GmbHG, 6. Aufl. 2009, \48 Rn. 25; Hüffer (o. Fn. 37), \48 Rn. 33.

51 Zutr. Roth, in: Roth/Altmeppen, GmbHG, 6. Aufl. 2009, $\$ 47$ Rn. 133.

52 Anders BGHZ 148, 335, 345 f.: Wiedergabe des für sich genommen eindeutigen Abstimmungsergebnisses im Protokoll genügt.

${ }^{53}$ Es ist umstritten, ob die bloße Einigkeit der präsenten Gesellschafter über das Beschlussergebnis einen mit der Anfechtungsklage anfechtbaren Beschluss darstellt (OLG München GmbHR 1990, 263 f.), oder ob hier mangels Beschlusses die Feststellungsklage gegeben ist; so zutr. Roth, in: Roth/Altmeppen, GmbHG, 6. Aufl. 2009, \47 Rn. 133. 
fassung ist ein Rechtshandeln. Es ist keine Überforderung von Gesellschaftern, die sich für ihre Geschäftstätigkeit der Rechtsform z.B. der GmbH bedienen, wenn man zur Beschlussfassung ein sauberes Verfahren mit Antragsstellung, Abstimmung und Beschlussfeststellung verlangt.

Wo der Ablauf der Versammlung protokolliert wird, ist neben den im Abstimmungsvorgang ermittelten Zahlen für „JA“, „NEIN“, „Enthaltung“ und „ungültig“ selbstverständlich auch und getrennt hiervon der anschließend festgestellte Beschlussinhalt zu protokollieren. Man kann nur fragen, ob die Protokollierung der Beschlussfeststellung ein Formerfordernis darstellt. Eine für alle Verbände gleichermaßen gültige Antwort lässt sich nicht geben; es ist auf die Rechtsgrundlagen, einschließlich der Satzung, zu verweisen. Eine nochmals andere Frage ist es, ob eine Beschlussfeststellung, die lediglich im Protokoll enthalten ist, in der Versammlung selbst aber nicht ausgesprochen worden ist, als Beschlussfeststellung zu gelten hat. ${ }^{54}$ Die Beschlussfeststellung ist nach der hier entwickelten Ansicht ein Konstitutivakt, der der Versammlung zugerechnet wird. Für die Setzung eines solchen Akts hat ein Protokollführer keine Zuständigkeit. Gegen den Willen des Versammlungsleiters - oder der Versammlung als ganzer, wo diese die Beschlussfeststellung übernimmt - kann der Protokollführer daher den Beschlussinhalt nicht aus eigenem Recht bestimmen. ${ }^{55}$ Auf Fragen der Beweiswirkung des Protokolls, die bei Divergenzen von tatsächlichem Versammlungsgeschehen und Protokollinhalt wichtig werden können, wird hier nicht eingegangen.

\section{Die Stimmabgaben in ihrem Verhältnis zum Beschluss}

Der Beschluss, der von einem Verbandsorgan gefasst wird, besteht nicht durch die abgegebenen JA-Stimmen in dem Sinne, dass deren Gesamtheit schon unmittelbar den Rechtsakt Beschluss darstellen würde. Der Positivbeschluss ist vielmehr als Rechtsakt von den in seinem Sinne abgegebenen JA-Stimmen verschieden, wie auch der Negativbeschluss nicht einfach in der Gesamtheit der - in sich unterschiedlichen - Stimmverhalten all derjenigen besteht, die zusammen die Annahme des Antrags verhindert haben. Der Beschluss ist ein ganzheitlicher Rechtsakt, er ist nicht aus den einzelnen Stimm-

${ }^{54}$ Hierzu (betr. Gesellschafterbeschlüsse der $\mathrm{GmbH}$ ): Roth, in: Roth/Altmeppen, GmbHG, 6. Aufl. 2009, $\ 48$ Rn. 25 f.

55 Anders könnte man allenfalls entscheiden, wenn das Protokoll von demjenigen herrührt, der für die Beschlussfeststellung zuständig ist, oder wenn der für die Beschlussfeststellung Zuständige die Beschlussfeststellung seitens des Protokollführers billigt, und nicht eine Kundgabe des Beschlussergebnisses an die Versammlung als Wirkform vorgeschrieben ist. 
abgaben „zusammengesetzt“ ${ }^{56}$ Die Stimmabgaben in ihrer Gesamtheit determinieren allerdings den Beschlussinhalt.

Die Fassung eines Beschlusses erfolgt in einem gestreckten Vorgang, in einem Verfahren. Zu diesem Verfahren gehört gleichermaßen die Ermittlung der Stimmen zur Antragsfrage wie die Beschlussfeststellung. ${ }^{57}$ Ein Stimmrechtsausschluss, der sich aus dem Beschlussgegenstand der Sachabstimmung ergibt, hindert den betroffenen Stimmberechtigten auch daran, an der Beschlussfeststellung teilzunehmen, weil die Beschlussfassung sich aus Sachabstimmung und Beschlussfeststellung ergibt. Die Beschlussfeststellung als Konstitutivakt schließt das Verfahren ab, soweit nicht noch zusätzliche Publizitätsakte nachfolgen müssen. Sieht man die Beschlussfeststellung als Konstitutivakt, so bildet das Gesamtergebnis der Stimmabgaben gleichsam den Sachverhalt, aufgrund dessen der Versammlungsleiter unter Beachtung der Regeln über Mehrheiten, Quoren, Gültigkeit und Auslegung von Stimmabgaben zu seiner Feststellung kommt, der Antrag, über den man abgestimmt hat, sei von der Versammlung angenommen oder abgelehnt. Die vorangehende Erzeugung des numerischen Abstimmungsergebnisses ist schon Teil des Vorgangs der Beschlussfassung. Sachabstimmung und Beschlussfeststellung bilden zusammen die Beschlussfassung, wobei die Beschlussfeststellung derart auf die Sachabstimmung bezogen ist, dass aus dem Abstimmungsergebnis der festzustellende Beschlussinhalt in gebundener Weise abzuleiten ist. Der Zusammenhang von Abstimmung und Beschlussfeststellung ist danach ein grundsätzlich anderer als nach der Vorstellung, derzufolge die einzelnen Stimmabgaben schon die Bestandteile des Rechtsgeschäfts „Beschluss" bilden, so dass mit abgeschlossener Stimmabgabe der Beschluss bereits gegeben sei. Einen Unterschied zwischen der Aktiengesellschaft und der $\mathrm{GmbH}$ oder dem Verein und den vereinsähnlichen Versammlungen (z.B. Versammlung der Wohnungseigentümer) ist in dieser Hinsicht nicht zu erkennen.

Da nach der hier zu entwickelnden Ansicht der Beschluss die Beschlussfeststellung zur konstitutiven Voraussetzung hat, kann jede einzelne Stimmabgabe nur auf dem Wege über eine Beschlussfeststellung wirksam werden.

${ }^{56}$ Es ist eine andere Frage, ob bei einem einzelnen Beschluss, in dem mehrere Beschlussgegenstände zusammengefasst sind, diese Beschlussgegenstände im Hinblick auf die Anfechtbarkeit oder Nichtigkeit ein getrenntes Schicksal haben können; s. dazu R. Schnorr, Teilfehlerhafte Gesellschafterbeschlüsse, 1997.

57 A.A. Zöllner (o. Fn. 7), S. 826 f. Die Annahme, die Beschlussfeststellung sei kein Teil der Beschlussfassung mehr, führt Zöllner zu der geradezu absurden Folgerung, Fehler bei der Ergebnisfeststellung seien keine Beschlussmängel (so S. 830); zur Richtigstellung bedürfe es einer eigenständigen, von der Anfechtungsklage verschiedenen „Ergebnisrichtigstellungsklage“. Mängel bei der Stimmauszählung sind idealtypische Beschlussmängel; dass Auszählungsfehler prozessual anders behandelt werden sollten als Ladungs- und Ankündigungsfehler, überzeugt nicht. 
Wenn es, anders als nach dem herkömmlichen Verständnis, zum Wirksamwerden der einzelnen Stimme nicht ausreicht, dass diese abgegeben ist, muss nach der hier vertretenen Ansicht der einzelne Stimmberechtigte kraft seines Stimmrechts einen Anspruch auf rechtlich einwandfreie Beschlussfeststellung haben. Dazu, wie dieser Anspruch auf Beschlussfeststellung vom einzelnen Stimmberechtigten klageweise durchgesetzt werden kann, wird noch Stellung genommen. ${ }^{58}$ Während nach von Tubr der Einfluss der Stimmenden auf die Beschlussfassung kategorial unterschiedlich ist, je nachdem, ob sie dem Antrag zustimmen oder nicht, ist nach der hier vertretenen Ansicht der Mitwirkungsbeitrag jedes Stimmenden zur Beschlussfassung gleich, indem jede Stimme, sei sie nun ein JA, ein NEIN oder eine Enthaltung, einen Beitrag zum numerischen Abstimmungsergebnis darstellt, auf dessen Grundlage der Beschluss festzustellen ist.

Nicht sachgemäß erscheint die Redeweise, wonach die Stimmabgaben in ihrer Gesamtheit den Beschluss "bewirken“. ${ }^{59}$ Die Stimmabgaben in ihrer Gesamtheit schaffen eine Situation, in der der Beschluss durch entsprechende Feststellung gefasst werden kann und muss, und sie geben jedem der Abstimmenden ein subjektives Recht auf eine diesbezügliche Feststellung: Der Beschluss wird durch die Stimmabgaben nicht bewirkt, aber bestimmt. Mit einem Ausdruck aus dem Prozessrecht ${ }^{60}$ könnte man sagen, die Stimmabgabe sei eine Erwirkungshandlung, aber keine Bewirkungshandlung. Die Stimmabgabe ist eine Verfahrenshandlung auf dem Gebiet des materiellen Rechts, aber nicht schon selbst die unmittelbare (Mit-)Setzung des materiell-rechtlichen Rechtsakts „Beschluss“.

Von Feine wurde die Ansicht vertreten, der Beschluss absorbiere die Stimmabgaben, so dass diese mit der Feststellung des Beschlusses „untergingen “ ${ }^{61}$ Es handelt sich um eine Zweckvorstellung, mit der Feine begründen wollte, warum eine eigenständige Anfechtung der Stimmabgabe gemäß SS $119 \mathrm{ff}$. BGB nach abgeschlossener Beschlussfassung nicht mehr möglich sein sollte. Die Frage der Anfechtung der Stimmabgabe sollte aber juristisch, d.h. als Interessenkonflikt, angegangen werden und nicht aufgrund einer Vorstellung wie der von der Absorption der Stimmabgaben im Beschluss. Zur Anfechtbarkeit der Stimmabgaben wird weiter unten noch Stellung genommen werden.

58 Unten XIII.

${ }^{59}$ Vgl. K. Schmidt (o. Fn. 11), S. 436: Beschluss gewinnt seine Wirksamkeit aus den Stimmerklärungen.

60 Vgl. Greger, in: Zöller, ZPO, 29. Aufl. 2012, vor \ 128 Rn. 14 m.w.N.

61 O. Fn. 9, S. 518. 


\section{Die Stimmabgabe als Erklärung im Rahmen des Abstimmungsvorgangs}

Zur Rechtsnatur der Stimmabgabe wird hier Stellung genommen von dem Ansatz aus, wonach die Stimmabgaben nicht schon zugleich die konstituierenden Bestandteile des Rechtsakts Beschluss sind, soweit es um die Beschlussfassung eines Verbandsorgans geht. ${ }^{62}$ Es ist für die hier vertretene Ansicht daher nicht entscheidend, ob die Stimmabgabe eine Willenserklärung ist ${ }^{63}$ oder nicht, weil der Beschluss als Rechtsakt zwar durch das Ergebnis der Abstimmung inhaltlich determiniert wird, aber nicht einfach in einer schlichten „Zusammensetzung“ aller Stimmabgaben (oder auch nur der Positivstimmen) besteht. Ob das Ergebnis des Stimmgangs aus einer Vielzahl von Willenserklärungen besteht oder aus einer Vielzahl von rechtsgeschäftsähnlichen Erklärungen, spielt für die hier verfolgte These keine entscheidende Rolle. Es erscheint wichtiger, konkret anzugeben, welche der für Willenserklärungen aufgestellten Gesetzesbestimmungen auf die Stimmabgabe anwendbar sind und welche nicht.

Die Stimmabgabe hat zum Inhalt, dass der Stimmende seine Stimmmacht einsetzt, um dem gestellten Antrag zur Annahme als Beschluss zu verhelfen (JA-Stimme) oder um dieser entgegenzuwirken (NEIN-Stimme). ${ }^{64}$ Nun zeichnet sich im Allgemeinen die (im eigenen Namen abgegebene) Willenserklärung dadurch aus, dass der Erklärende für sich eine Regelung setzt, an die er gebunden ist. Die Stimmabgabe unterscheidet sich von den Willenserklärungen dieser Art, weil der Stimmende damit nicht an der Setzung einer ibn selbst betreffenden Regelung mitwirkt. Charakteristisch für die Stimmabgabe ist vielmehr, dass der Stimmende mit seiner Erklärung hinsichtlich eines Entschlusses wirkt, der Geltung nicht für ihn selbst, sondern für den Verband erlangt. Das Stimmrecht der Mitglieder ist, wie es in den Motiven zum BGB für den Verein heißt, „die Befugnis, bei der Herstellung des Willens für die Körperschaft mitzuwirken“.65 Wie wir gesehen haben, ${ }^{66}$ „bewirkt“ die Stimmabgabe bei einer Abstimmung in einem Verbandsorgan für sich genommen rechtlich nur, dass bei der anschließenden Beschlussfeststellung diese Stimmabgabe zutreffend erfasst und für die Ermittlung des Abstimmungsergebnisses berücksichtigt werden muss.

${ }^{62}$ S. oben II zur Beschlussfassung ohne Organbezug, z.B. durch Bruchteilseigentümer.

${ }^{63}$ So die ganz herrschende Ansicht; s. statt aller K. Schmidt (o. Fn. 11), S. 437; Jacoby

(o. Fn. 11), S. 421; zuletzt Dröge (o. Fn. 13), S. 18 mit umf. Nachw. in Fn. 30; Winnefeld

(o. Fn. 26) sprach konsequent der NEIN-Stimme die Qualität einer Willenserklärung ab.

${ }^{64}$ S. auch W. Ernst (o. Fn. 48), Rn. 61.

${ }^{65}$ Motive I, 107 = Mugdan I, 411.

${ }^{66}$ Soeben VI. 
Inwieweit die Vorschriften, die das BGB für die Willenserklärung aufstellt, auf die Stimmabgabe angewendet werden können, sei anhand eines Durchgangs durch die einzelnen Sachfragen geprüft: ${ }^{67}$

(a) Die einzelne Stimmabgabe ist ein einseitiger Vorgang, so dass sich von den Vorschriften aus dem Allgemeinen Teil des BGB diejenigen zur analogen Anwendung anbieten, die das einseitige Rechtsgeschäft betreffen.

(b) Die Stimmabgabe muss in der Weise erfolgen, die durch Gesetz oder Gesellschaftervertrag/Satzung, eine Versammlungsordnung oder seitens des Versammlungsleiters (eventuell auch durch einen Ordnungsbeschluss der Versammlung) festgesetzt ist. Wenn die Festlegung durch Gesetz erfolgt ist, kann man für die Ungültigkeit einer Stimmabgabe, die der gesetzlichen Bestimmung nicht entspricht, auf $\mathbb{1 2 5} \mathrm{S} .1 \mathrm{BGB}$ verweisen; liegt eine Satzungsbestimmung zugrunde, mag $\mathbb{\$} 125 \mathrm{~S}$. 2 BGB analog angeführt werden. Man muss aber auch eine Stimmabgabe für ungültig halten, die nicht der vom Versammlungsleiter (oder in einem Ordnungsbeschluss) getroffenen Festlegung entspricht. Wenn die Abstimmung z.B. schriftlich erfolgt, muss eine durch Zuruf erfolgte Stimmabgabe für ungültig erachtet werden. Wenn man meinen würde, hierfür bedürfte es eines positivistischen Anhaltspunktes, könnte man ebenfalls auf den Rechtsgedanken des $\ 125$ S. 2 BGB verweisen.

Von den Formerfordernissen, die für den Beschluss aufgestellt sind (z.B. \130 I $1 \mathrm{AktG}$ ), wird angenommen, dass sie nur für den Akt der Beschlussfeststellung gelten: Wenn für den konkreten Beschluss eine notarielle Beurkundung vorgeschrieben ist, bedeutet dies gerade nicht, dass auch die Abgabe der einzelnen Stimme notariell beurkundet werden müsste. Noack hat in diesem Zusammenhang davon gesprochen, dass sich die Formbedürftigkeit eben nicht auf „Vorstufen“ des Rechtsgeschäfts beziehe. ${ }^{68}$ Auch die einzelne Stimmabgabe muss in diesem Sinne als „Vorstufe“ zum Beschluss gesehen werden, die als solche, obschon Teil des Verfahrens der Beschlussfassung, von einem etwaigen Formerfordernis für den Beschluss nicht erfasst ist. Schon für sich genommen sollte diese Handhabung des Formerfordernisses für den Beschluss ausreichen, die Ansicht von der Identität des Beschlusses mit der Gesamtheit der zustimmenden Stimmabgaben zu widerlegen: Bei einem mehrseitigen Rechtsgeschäft, etwa bei einem Auseinandersetzungsvertrag über ein Grundstück, erstreckt sich ein Formerfordernis auf die einzelnen Willenserklärungen, womit dem Formerfordernis dann auch Genüge getan ist. Für den formbedürftigen Beschluss der Hauptversammlung - m.E.

${ }^{67}$ Die folgende Darstellung untersucht die Stimmabgabe in der Versammlung; es wird davon ausgegangen, dass die Stimmabgabe, wenn sie in Zirkularverfahren erfolgt, dadurch ihre Rechtsnatur nicht grundlegend verändert.

${ }^{68}$ O. Fn. 23, S. 31. 
aber für den Beschluss von Organen der Verbände des Privatrechts überhaupt - kann die Vorstellung vom mehrseitigen, aus Stimmabgaben aggregierten Rechtsgeschäft keinesfalls zutreffen.

(c) Eine Willenserklärung ist empfangsbedürftig, wenn der Erklärende den Zugang seiner Erklärung bei einer empfangszuständigen Person bewirken muss. Der Stimmende muss die Stimmabgabe in der Weise vornehmen, die vom Versammlungsleiter vorgegeben worden ist, sei dies das Handheben, das Heben von Stimmkarten, das Ausfüllen von Stimmzetteln, das Drücken von Schaltern oder dergleichen. Es ist dann Sache des Versammlungsleiters, alle ordnungsgemäß erfolgenden Stimmabgaben wahrzunehmen und zu erfassen; er ist insoweit zu einem aktiven Ordnungshandeln verpflichtet. Ohne Rücksicht auf einen Zugang ist jede Stimme zu erfassen, die im Einklang mit den angeordneten Modalitäten abgegeben worden ist. Demgegenüber kann der Zugang einer Willenserklärung herkömmlicherweise auch gegenüber einem passiven Erklärungsempfänger erfolgen. Der Vorgang von Abgabe und Erfassung der Stimme wird mittels der Begriffe Zugang und Empfangszuständigkeit nicht zutreffend erfasst. ${ }^{69}$

(d) Die Stimmabgabe kann nur in einem bestimmten, kurzen Zeitraum erfolgen, nämlich auf den Aufruf zur Stimmabgabe hin. Die Abstimmung ist ein Präsenzgeschäft. Vorgängige (briefliche) oder nachträgliche Stimmabgaben sind grundsätzlich nicht möglich.

(e) Die Stimmabgabe hat das JA oder das NEIN zum Antrag zum möglichen Inhalt. Die Stimmabgabe kann nur ausdrücklich erfolgen, nicht durch schlüssiges Verhalten. ${ }^{70}$ In der technischen Ausgestaltung des Abstimmungsvorgangs wird man bemüht sein, die Stimmabgabe so zu regeln, dass Auslegungszweifel von vornherein ausgeschlossen werden. Es ist misslich, wenn bei schriftlicher Stimmabgabe die Frage auftritt, ob ein "Sehr gut" auf dem Stimmzettel als ein JA genommen werden muss. Daher legt man die Stimmenden zweckmäßig auf den Gebrauch der Worte JA und NEIN fest oder sorgt auf andere Weise für ein möglichst eindeutiges Stimmverhalten, etwa derart, dass die Stimmkarte bei JA in eine bestimmte Urne geworfen wird, bei NEIN in eine andere o.ä. Sofern aber Stimmabgaben auslegungsbedürftig bleiben, kann $\ 133$ BGB angewendet werden.

(f) Die Stimmabgabe ist eine Rechtsausübung. Die Gültigkeit der Stimmabgabe hängt davon ab, dass durch sie ein bestehendes Stimmrecht ausgeübt wird. Die Ausübung des Stimmrechts führt sodann, bezogen auf den betreffenden Stimmgang, zu dessen Erschöpfung: Niemand kann seine Stimme in ein und derselben Abstimmung „doppelt“ abgeben. Dem entspricht es, dass

\footnotetext{
69

Flume (o. Fn. 14), S. 249: „Es gibt hinsichtlich der Stimmabgabe überhaupt keinen Erklärungsgegner."

70 W. Ernst (o. Fn. 48), Rn. 137.
} 
die Stimmabgabe unwiderruflich erfolgt. ${ }^{71}$ Mit der Stimmabgabe hat sich der Stimmende bereits selbst gebunden; die Bindung kraft des Beschlusses setzt dagegen erst nach dessen Feststellung ein. Gerade wenn man die Qualifizierung der Stimmabgabe als Willenserklärung befürwortet und ernst nimmt, verbietet sich die Zulassung eines Widerrufs. Weiterhin ist die Stimmabgabe bedingungsfeindlich.

Mit der Stimmabgabe übt der Stimmberechtigte ein ibm selbst zustehendes Recht aus; er handelt nicht als Vertreter des Verbandes oder der Versammlung. Das Problem eines eventuellen Konflikts zwischen der mitwirkenden Herstellung des Willens des Verbandes und eventuellen Eigeninteressen des Stimmberechtigten wird dabei abschließend durch die Regelungen zum Stimmrechtsausschluss bewältigt; $\int 181$ BGB findet auf die Stimmabgabe insofern keine Anwendung. ${ }^{72}$ Der Anwendung des $\ 181$ BGB steht nach dem hier vertretenen Ansatz schon entgegen, dass die Stimmabgabe kein Handeln zur Vornahme eines „Rechtsgeschäfts“ ist, da die einzelne Stimmerklärung nicht zum Bestandteil des Rechtsgeschäfts „Beschluss“ wird.

(g) Die Frage der Stimmfähigkeit kann anhand der $\$ \int 104$ ff. BGB beurteilt werden. Der beschränkt geschäftsfähige Stimmrechtsinhaber ${ }^{73}$ bedarf zur Ausübung des Stimmrechts der Einwilligung des gesetzlichen Vertreters; $\int 107$ BGB analog. Das Vorliegen dieser Einwilligung ist bei der Stimmrechtsprüfung festzustellen; kann sich der Versammlungsleiter nicht vom Vorliegen der Einwilligung überzeugen, lässt er den Minderjährigen nicht mitstimmen. $\mathrm{Zu}$ einer Zurückweisung der Stimmabgabe analog \ 111 S. 2 BGB sollte es von daher nicht kommen. Eine Genehmigung der ohne Einwilligung abgegebenen Stimme kommt nicht infrage; sie wäre auch mit dem Charakter der Abstimmung als Präsenzgeschäft unvereinbar.

Zweifelhaft ist die Anwendung des $\ 107$ BGB hinsichtlich des Begriffs des rechtlichen Vorteils. Bei der Stimmabgabe handelt es sich um die Ausübung der „Befugnis, bei der Herstellung des Willens für die Körperschaft mitzuwirken“. ${ }^{74}$ Es verhält sich deswegen aber nicht so, dass die Stimmabgabe zwangsläufig ein neutrales Geschäft wäre, das vom beschränkt geschäftsfähigen Minderjährigen ohne Einwilligung seines gesetzlichen Vertreters vorgenommen werden könnte. Beschlüsse können die Rechtsstellung des Stimmenden durchaus unmittelbar beeinflussen; man denke an den Beschluss über eine Erhöhung von Beiträgen, über die Feststellung der Dividende und

${ }^{71}$ Die Widerruflichkeit der Stimmabgabe ist umstritten; s. K. Schmidt (o. Fn. 11), S. 437 m.w.N.; BGH NJW-RR 1990, 798 betraf einen Sonderfall.

${ }^{72}$ Flume (o. Fn. 14), S. 237; ausf. J. Wilhelm, JZ 1976, 674 ff.; a.A. zu den Gesellschafterbeschlüssen der GmbH Zöllner, in: Baumbach/Hueck, GmbHG, 19. Aufl. 2010, $\mathbb{4} 47$ Rn. $60 \mathrm{ff}$.

${ }^{73}$ Zum minderjährigen Aktionär (aus Sicht des schweiz. Aktienrechts) instruktiv N. P. Vogt/D. Leu, Festschrift Rolf Watter, 2008, S. 449, 452 ff.

${ }^{74}$ Motive I, 107 = Mugdan I, 411. 
dergleichen. Bei solchen Beschlüssen würde die Möglichkeit des beschränkt Geschäftsfähigen, seine Stimme ohne Zustimmung des gesetzlichen Vertreters abzugeben, davon abhängen, ob der Beschluss für ihn als rechtlich lediglich vorteilhaft angesehen werden kann. Eventuell käme man sogar dazu, dass für den Minderjährigen die Abgabe einer JA- (oder NEIN-)Stimme als rechtlich lediglich vorteilhaft anzusehen wäre, nicht jedoch die Abgabe der Stimme im gegenteiligen Sinne. Wenn die Gültigkeit der Stimmabgabe von deren Inhalt abhängig wäre, könnte von einer freien Ausübung der „Befugnis, bei der Herstellung des Willens für die Körperschaft mitzuwirken,“ keine Rede mehr sein. Es erscheint besser, das Konzept des rechtlich lediglich vorteilhaften Geschäfts auf die Stimmabgabe überhaupt nicht anzuwenden. Sollen beschränkt Geschäftsfähige ihr Stimmrecht selbst ausüben, ist daher die Einwilligung des gesetzlichen Vertreters unverzichtbar.

(h) Wo das Stimmrecht nicht höchstpersönlicher Natur ist, kann die Stimmabgabe durch einen Stellvertreter erfolgen. Hierfür stellen die $\mathbb{S} 164 \mathrm{ff}$. BGB eine im Grundsatz brauchbare Normgrundlage dar. Die Bevollmächtigung muss aber auf den Vorgang der Stimmrechtsprüfung abgestimmt werden. Da das Vorliegen einer Vollmacht bei der Stimmrechtsprüfung festzustellen ist, scheidet eine interne Bevollmächtigung ( $\$ 167$ I 2. Alt. BGB) aus. Eine konkludente Bevollmächtigung wird man nicht zulassen, weil sie keine Grundlage für die Stimmrechtsprüfung bietet. Kann sich der Versammlungsleiter bei der Stimmrechtsprüfung nicht vom Vorliegen der Vollmacht überzeugen, lässt er den Vertreter nicht mitstimmen. Zu einer Zurückweisung der Stimmabgabe analog $\mathbb{1} 174$ BGB braucht es von daher nicht zu kommen.

Es ist nicht möglich, einen vollmachtlosen Vertreter mitstimmen zu lassen, so dass der Stimmberechtigte die Stimmabgabe anschließend genehmigt; hierfür kann man sich auf $\$ 180$ S. 1 BGB stützen. Gelegentlich wird vorgeschlagen, man könne vollmachtlose Stimmrechtsvertreter unter den Voraussetzungen des $₫ 180$ S. 2 BGB mitstimmen lassen. ${ }^{75}$ Dem steht nicht nur das Rechtsproblem der fehlenden Empfangszuständigkeit für die Stimmabgabe entgegen (oben c). Eine Pendenz der Stimmabgabe nach $\$ \$ 177,180$ S. 2 BGB verbietet sich wegen der Untrennbarkeit von Stimm- und Rederecht: Der vollmachtlose Vertreter könnte in der Versammlung das Wort ergreifen und die Aussprache beeinflussen. Diese Einflussnahme wäre nicht ungeschehen zu machen, wenn der Stimmrechtsinhaber schließlich die Genehmigung des vollmachtlosen Handelns verweigert. Eine vollmachtlose Teilnahme nach $\$ 180$ S. 2 BGB wäre also allenfalls dergestalt denkbar, dass sich der vollmachtlose Vertreter bloss zur Abgabe der Stimme, unter Ausschluss von Rede- und Antragsrecht, einfindet; eine derartige Rechtsaufspaltung des Teilnahmerechts wird man für die meisten Verbände ablehnen.

75 C. Hartmann, DNotZ 2002, 253 ff. (für die AG); vorsichtiger M. Häublein, ZWE 2012, 1, 11 f. (für die Versammlung der Wohnungseigentümer). 
Beim Stimmvertreter können Eigeninteressen bestehen, die es zweifelhaft erscheinen lassen, ob er das fremde Stimmrecht pflichtgemäss ausüben wird. Indem die gesetzlichen Regelungen zum Stimmrechtsausschluss nach $\ 136$ I AktG, $\mathbb{\int} 47$ IV GmbHG auch die Ausübung eines fremden Stimmrechts erfassen („für sich oder einen anderen“), führt auch ein in der Person des Stimmvertreters gegebener Ausschlussgrund zum Stimmrechtsausschluss. Damit besteht eine abschließende Regelung, die für die zusätzliche Anwendung des $\int 181$ BGB wohl keinen Raum lässt. Sofern der Stimmrechtsausschluss weder durch Gesetz noch durch Satzung geregelt ist, ${ }^{76}$ liegt eine analoge Anwendung der positivrechtlichen Regelungen zum Stimmrechtsausschluss näher als eine Anwendung des $\int 181 \mathrm{BGB}:{ }^{77} \mathrm{Nach}$ dem hier vertretenen Ansatz ist die Stimmabgabe kein Handeln zur Vornahme eines „Rechtsgeschäfts“, weil die einzelne Stimmerklärung nicht zum Bestandteil des Rechtsgeschäfts „Beschluss“ wird, und daher könnte es sich auch hinsichtlich $\ 181$ BGB von vornherein nur um eine analoge Anwendung handeln. Vorzugwürdig erscheint demgegenüber die (gesamt-)analoge Anwendung von Vorschriften über den Stimmrechtsausschluss, die es auch besser ermöglichen, im Rahmen der Stimmrechtsprüfung der Abgabe der fraglichen Stimme schon präventiv entgegenzutreten. Die Frage wäre eine Vertiefung wert.

(i) Da es sich bei der Stimmabgabe um eine Rechtsausübung handelt, kann man sich auch fragen, ob eine Ermächtigung dergestalt möglich sei, dass der Ermächtigte das Stimmrecht im eigenen Namen ausübe; als normative Grundlage könnte $\ 185$ I BGB angeführt werden. ${ }^{78}$ Da die Ermächtigung bei der Stimmrechtsprüfung nachgewiesen werden müsste, dürfte sich ein interessanter Unterschied zur Stimmrechtsvertretung nicht ergeben. Die Frage wird hier nicht vertieft.

(j) Die Frage, ob die Stimmabgabe wegen Willensmängeln angefochten werden kann, ist schon Gegenstand einer umfangreichen Kontroverse gewesen. Ausführlich wurde die Frage von Bartholomeyczik behandelt. ${ }^{79} \mathrm{Da}$ die Stimmabgabe selbstbestimmt erfolgt, ist heute anerkannt, dass man sich von einer durch Irrtum, Täuschung oder Drohung bewirkten Stimmabgabe muss „lossagen“ können. ${ }^{80}$ Es können zum Schutz der Selbstbestimmung die Vorschriften entsprechend angewendet werden, die bei Irrtum, Täuschung

${ }^{76}$ So verhält es sich bei $\$ 25$ WEG; dazu Häublein (o. Fn. 75), S. $14 \mathrm{f}$.

77 Häublein weist zutr. darauf hin, dass $\ 181$ BGB die unpassende Möglichkeit der Gestattung des Insichgeschäfts einschliesst, (o. Fn. 75), S. 14 Fn. 122.

$78 \mathrm{Vgl}$. für die Aktiengesellschaft $\$ 129$ III AktG.

79 H. Bartholomeyczik, AcP 144 (1938), 287 ff.; ausf. auch Baltzer (o. Fn. 7), S. 152 ff.

${ }^{80}$ Legt man den von Tubr'schen Beschlussbegriff zugrunde, demzufolge nur die zustimmenden Stimmerklärungen den Beschluss ausmachen, käme die Möglichkeit einer Anfechtung der Stimmabgabe wegen Willensmängeln streng genommen auch nur für diejenigen in Betracht, die dem Antrag zugestimmt haben. 
oder Drohung die Anfechtung einer Willenserklärung erlauben. Während der Versammlung erfolgt die Anfechtung gegenüber dem Versammlungsleiter; nach Abschluss der Versammlung gegenüber demjenigen, der den Verband vertritt. ${ }^{81}$ Die Anfechtbarkeit der Stimmabgabe ist nicht nur dann zuzulassen, wenn damit mittelbar der Beschluss zu Fall gebracht werden könnte. Schon weil der Stimmende u.U. für seine Stimmabgabe haftbar gemacht werden kann, ${ }^{82}$ darf er nicht an eine mit einem Willensmangel behaftete Stimmabgabe gebunden sein. Auf die Anfechtung der Stimmabgabe ist nochmals zurückzukommen. ${ }^{83}$

Mit den vorstehenden Einzelheiten dürfte die Stimmabgabe als Rechtshandeln im Wesentlichen beschrieben sein. Wie soll man nun die Stimmabgabe kategorial erfassen? Soweit ein Rechtsgeschäft durch mehrere Willenserklärungen gebildet wird, die Willenserklärungen also Teil des rechtsgeschäftlichen Tatbestands sind, knüpfen sich die Rechtswirkungen nicht an die Willenserklärung als solche, sondern an das Rechtsgeschäft. ${ }^{84}$ Beachtet man die Differenz von Willenserklärung und Rechtsgeschäft, so ist die einzelne Stimmabgabe sicherlich kein Rechtsgeschäft, weil durch die isolierte Stimmabgabe eine rechtliche Regelung nicht gesetzt wird. Ist die Stimmabgabe eine Willenserklärung? Nach den Vorschriften des BGB über Willenserklärungen kann die Stimmabgabe behandelt werden, sofern zugleich darauf Rücksicht genommen wird, dass die Stimmabgabe eine in den Abstimmungsvorgang eingebettete Erklärung ist, die den Vorgaben für das Abstimmungsverfahren entsprechen muss: Die Stimmabgabe kann wegen ihrer Einbettung in das Abstimmungsverfahren nur zu einem ganz bestimmten Zeitpunkt, in einer ganz bestimmten Weise und mit einem von zwei fixen Inhalten (JA oder NEIN) erfolgen. ${ }^{85}$ Ebenso muss bei Einsatz eines Stimmvertreters dieser so bevollmächtigt werden, dass der Vertreter bei der Stimmrechtsprüfung als stimmberechtigt ermittelt werden kann. Diese und weitere, durch den Verfahrensbezug gegebene Rahmenbedingungen für die Stimmabgabe schließen nicht aus, dass man auch Vorschriften des BGB über Willenserklärungen auf die Stimmabgabe anwendet, wobei aber die üblichen Gestaltungsmöglichkeiten des Erklärenden, seine Willenserklärung inhaltlich und durch Wahl von Ausdrucksmittel und Erklärungszeit zu bestimmen, durch die mit dem Verfahrensbezug gegebenen Vorgaben stark eingeschränkt sind. Die Einbettung in den Verfahrensbezug ändert aber nichts daran, dass mit dem JA oder

${ }^{81} \mathrm{Vgl}$. Flume (o. Fn. 14), S. 250.

${ }^{82}$ Dazu umf. Dröge (o. Fn. 13).

${ }^{83}$ S. unten XV.

${ }^{84}$ S. W. Flume, Das Rechtsgeschäft, 4. Aufl. 1992, S. 25 ff.; D. Leenen, Festschrift Canaris, 2007, Bd. I, S. $699 \mathrm{ff}$.

${ }_{85}$ Die Erhaltung ist als eine erklärte Nichtausübung des Stimmrechts zu erfassen; s. Ernst (o. Fn. 48), Rn. 62 ff. 
NEIN ein Wille kundgetan wird, der als psychologische Tatsache existiert, und zwar dazu, welches der Gesamtwille des Verbandes sein soll, und dass weiterhin die Abgabe der Stimme nicht fremd-, sondern selbstbestimmt erfolgt, wobei es aber darum geht, was für den Verband gelten soll. Am ehesten ist in diesem Umstand, dass der Stimmberechtigte autonom über die Stimmabgabe entscheidet, die Ähnlichkeit zur Willenserklärung des BGB begründet. Die Stimmabgabe ist danach eine Verfahrenshandlung im Abstimmungsvorgang, für deren rechtliche Beurteilungen Vorschriften des BGB über die Willenserklärungen entsprechend angewendet werden können, wobei auf den Verfahrensbezug und -zweck der Stimmabgabe Rücksicht zu nehmen ist. Folgt man der hier entwickelten Ansicht, dann liegt für die Stimmabgabe näher als der Vergleich mit der Willenserklärung der Vergleich mit der Prozesshandlung, wobei es sich natürlich bei der Abstimmung nicht um ein gerichtliches Verfahren handelt, sondern um ein Verfahren auf dem Gebiet des materiellen Rechts. Wie man sich sehr zurückhält, die Prozesshandlung im Zivilprozess nach den Vorschriften des BGB über Willenserklärungen zu behandeln ${ }^{86}$ sollte man auch bei der Stimmabgabe als einer Verfahrenshandlung des materiellen Rechts von einer voreiligen einschränkungslosen Gleichsetzung mit der Willenserklärung absehen.

Unabhängig davon, ob man die Stimmabgabe als Willenserklärung ansieht, ist jedenfalls die begrenzte, von der Stimmabgabe ausgehende Rechtswirkung zu beachten: Die abgegebene Stimme hat in die Ermittlung des Abstimmungsergebnisses einzugehen und bildet - zusammen mit allen anderen abgegebenen Stimmen - die gleichsam "tatbestandliche“ Grundlage für den Beschluss. Die Stimmabgabe hat insofern "Tatbestandswirkung“ für die Beschlussfeststellung. In diesem Beitrag zur Bildung des Tatbestandes für die Beschlussfeststellung erschöpft sich die Rechtswirkung der Stimmabgabe. Als Verfahrenshandlung ist die Stimmabgabe eine „Erwirkungshandlung“. Die vom Beschluss ausgehenden Rechtsfolgen beziehen ihre Geltung dagegen nicht aus den Stimmerklärungen, mag man diese auch weitgehend wie Willenserklärungen behandeln: Die rechtlichen Beschlussfolgen sind nicht Rechtswirkungen, die unmittelbar deswegen eintreten, weil sie mittels Stimmabgaben als „gewollt“ erklärt worden sind.

\section{Die Geltung (Bindungswirkung) des Beschlusses}

Der Beschluss hat zum Inhalt, dass die Versammlung - für den Verband in rechtlich einwandfreier Weise einen bestimmten Entschluss („Willen“) gefasst hat. Der Beschluss zeitigt eine materielle Bindungswirkung. Diese

${ }^{86}$ S. statt aller D. Schwab, Festschrift Baumgärtel, 1990, S. 503 ff. 
besteht darin, dass der Verband, seine Mitglieder und Organe, den Beschluss, wenn er aktuell wird, respektieren. Mitglieder und Organe müssen den Beschluss ihrem diesbezüglichen Handeln zugrunde legen, und zwar - wie zu zeigen sein wird - unabhängig davon, ob der Beschluss rechtmäßig oder rechtswidrig ist. Die Bindungswirkung des Beschlusses ist eine Folge der innerverbandlichen Zuständigkeitsordnung, die es der Versammlung erlaubt, in bestimmten Fragen und in einem bestimmten Verfahren den für den Verband maßgeblichen Willen zu generieren. ${ }^{87}$

Der Akt der Beschlussfassung ist als solcher rechtlich belastbar. Wenn der Beschluss durch weitere Aktivitäten umzusetzen ist und hierfür auf die Willensbildung des Verbandes Bezug genommen werden muss, dann genügt der Verweis auf den Beschluss. Wenn etwa von den Mitgliedern höhere Beiträge eingefordert werden, die in der Versammlung beschlossen worden sind, genügt die Berufung auf den Beschluss, um eine schlüssige Geltendmachung des Anspruchs zu erlauben und ein zahlungsunwilliges Mitglied in Verzug zu setzen. Typischerweise wird der Beschluss durch einen Auszug aus dem Protokoll nachgewiesen. Um die Forderung nach den erhöhten Mitgliedsbeiträgen in einer verzugsbegründenden Weise geltend zu machen, ist es nicht erforderlich, jedem einzelnen Mitglied die für die fragliche Beschlussfassung abgegebenen Stimmzettel vorzuweisen und ihm zu erläutern, welcher Beschlussinhalt sich daraus ergibt; man muss auch nicht dartun, dass alle Stimmberechtigten ordnungsgemäß einberufen worden waren, Stimmrechtsausschlüsse beachtet wurden und dergleichen mehr. ${ }^{88}$ Es kann gar nicht anders sein, als dass der Verband und seine Mitglieder an den Beschlussinhalt gebunden sind, den die Versammlung - durch ihren Leiter oder durch eigene Ordnungsentscheidung - festgestellt hat. Es wäre sinnlos, die Versammlung feststellen zu lassen, sie habe so und so beschlossen, wenn man diese Feststellung anschließend rechtlich nur dergestalt beanspruchen könnte, dass man bei jedem auf den Beschluss gestützten Rechtshandeln stets von neuem die Rechtmäßigkeit der Beschlussfassung in allen ihren Einzelheiten dartäte und dazu auf die einzelnen Stimmabgaben zurückkäme.

Der Beschluss ist ein Konstitutivakt ${ }^{89}$ mit inhärenter Bindungswirkung. Die Bindungswirkung des Beschlusses ist dem Aktstyp „Beschluss“, jeden-

${ }^{87}$ Die Bindungswirkung betrifft auch die Versammlung selbst, indem ein Rückkommen auf den bereits gefassten Beschluss nicht ohne weiteres zulässig ist; s. Ernst (o. Fn. 48), Rn. $273 \mathrm{ff}$.

${ }_{88}$ Wenn umgekehrt eine Beschlussfeststellung zu der Abstimmung über die Mitgliedsbeiträge unterblieben ist, nachdem alle Stimmzettel in einer Urne eingesammelt worden waren, ist es nach dem hier vertretenen Ansatz nicht möglich, gegenüber dem einzelnen Mitglied den Anspruch auf die Beiträge geltend zu machen, indem man ihm die Urne mit den Stimmzetteln präsentiert und erklärt, die Mehrheit habe, wie jeder nachzählen könne, dafür gestimmt.

${ }^{89}$ Der Ausdruck geht zurück auf Feine (o. Fn. 9), S. 517 u. öfter. 
falls soweit er in organisierten Verbänden gefasst wird, eigen; sie hängt nicht weiter von der Rechtsform des Verbandes ab. Die Bindungswirkung des Beschlusses beruht auch nicht erst darauf, dass das spezifische Verbandsrecht das prozessuale Vorgehen gegen den Beschluss regelt und beschränkt. Es entspricht vielmehr dem Sinn der Beschlussfassung, dass der am Ende stehende Willensentschluss für den Verband „gilt“ und dass für die spätere Berufung auf diesen Willensentschluss ein Zurückgehen auf die Einzelheiten der Beschlussfassung entbehrlich ist. Die Bindungswirkung ist eine echte Rechtswirkung des Beschlusses. Die Beschlussfassung ist ein Akt der Setzung. Die Konstitutivwirkung des Beschlusses ist darin begründet, dass die hierzu autorisierte Versammlung mit dem Beschluss feststellt, sie habe mit der rechtlich erforderlichen Mehrheit einen bestimmten Antrag angenommen und damit zum „Gesamtwillen“ des Verbandes erhoben. Von der Bindungswirkung des Beschlusses her, ohne die der Aktstyp „Beschluss“ nicht sinnvoll wäre, wird noch einmal deutlich, dass die Beschlussfassung nicht schon mit der Stimmabgabe aller Anwesenden abgeschlossen sein kann. Vom Standpunkt des von Tubr'schen Beschlussbegriffes aus ist ja kaum zu erklären, wie der Beschluss die ihm eigene Bindungswirkung entfalten kann: Ein zu den tragenden Stimmabgaben hinzutretender, überpartikularer Akt wie die Beschlussfeststellung wird ja für entbehrlich gehalten; wo er vorkommt, wird ihm eine zusätzliche Rechtswirkung im Grundsatz abgesprochen. Eine Bindungswirkung, die das Zurückgehen auf die Gesamtheit der Stimmabgaben entbehrlich macht, kann aber überhaupt nur entstehen, wenn der Beschluss überpartikular festgestellt wird. All dies gilt beileibe nicht nur für die Aktiengesellschaft und die Beschlüsse ihrer Hauptversammlung, sondern ebenso für die $\mathrm{GmbH}$ und den Verein und die vereinsähnlich agierenden Gemeinschaften.

\section{Die Abhängigkeit des Beschlusses vom Verbandsrecht}

In der Diskussion um den Beschlussbegriff war es ein besonderes Verdienst Philipp Hecks, darauf hingewiesen zu haben, dass der Beschluss als Rechtsakt nicht isoliert gedacht werden kann. Vielmehr erfolge jede Beschlussfassung, so Heck, aufgrund einer „Grundrelation“, die durch den Verband und seine Verfassung gegeben sei. ${ }^{90}$ Der Beschluss sei insoweit ein unselbständiges Geschäft, als er nur in der Einbettung in das Verbandsrecht

90 P. Heck, Festschrift Otto v. Gierke, 1911, S. 319 ff.; bei Heck werden auch die weiteren zeitgenössischen Ansätze zur Erfassung des Beschlusses besprochen, insb. S. 354 ff. auch die Redeweise vom „Gesamtakt“, die zurückgeht auf $J$. E. Kuntze, Festgabe Leipziger Juristenfakultät f. O. Müller, 1892, S. 27 ff. Ablehnend zur Lehre vom Gesamtakt Flume (o. Fn. 84), S. 602 f. 
vorkomme. Hieran soll nun angeknüpft werden. Beschlussfassung setzt eine rechtlich begründete Beschlussfassungskompetenz voraus, eine Zuständigkeit. Jeder Beschluss wird als „abhängiger“ Rechtsakt auf der Grundlage einer verbandsrechtlichen Organisations- und Zuständigkeitsverfassung gefasst. Anschaulich hat Ulrich Noack von der „Ermächtigungsgrundlage“ für den Beschluss gesprochen. ${ }^{91}$ Der Beschluss muss entsprechend den Zuständigkeits- und Verfahrensregelungen, die im und für den Verband gelten, zustande gebracht werden. ${ }^{92}$ Die Abhängigkeit des Beschlusses von der - wie Heck es nannte - "Grundrelation“ wird von Dröge jetzt im Anschluss an Baltzer ${ }^{93}$ als ein „Zurechnungsproblem“ angesprochen: Nur wenn die Beschlussfassung im verbandsverfassungsrechtlich zuständigen und korrekt konstituierten, zudem beschlussfähigen Organ unter Einhaltung der vorgeschriebenen Förmlichkeiten erfolge, werde der Beschluss dem Verband zugerechnet. ${ }^{94}$ In unterschiedlichen Wendungen wird hier treffend ein grundlegendes Charakteristikum des Beschlusses - zugleich das Unterscheidungsmerkmal zum „unabhängigen“ Rechtsgeschäft - ausgemacht.

\section{Rechtmäßigkeit und Rechtswidrigkeit von Beschlüssen}

Die Abhängigkeit des Beschlusses als Rechtsakt, der Umstand, dass ein Beschluss nur im Rahmen einer Verbandsordnung gefasst werden kann, ist die Ursache dafür, dass für den Beschluss eine Frage gestellt werden kann, zu der es bei gewöhnlichen („unabhängigen“) Rechtsgeschäften kein Gegenstück gibt: Wie wirkt es sich rechtlich auf den Beschluss aus, wenn die für die Beschlussfassung bestehenden normativen Vorgaben nicht beachtet wurden? Die Abhängigkeit des Beschlusses als Rechtsakt ist der Ursprung der Lehre von den Beschlussmängeln: Weil der Beschluss in Abhängigkeit von einer Vielzahl verbandsverfassungsrechtlicher Normen gefasst wird - und auch nur so gefasst werden kann -, stellt sich eben die Frage nach den Folgen einer Verletzung derjenigen Normen, welche die Grundlage für die Beschlussfassung - deren „Ermächtigungsgrundlage“ - darstellen. Es kann hier nicht

91 O. Fn. 23, S. 55.

92 Die Stimmberechtigten sind einwandfrei einzuberufen - die Stimmberechtigungen müssen geprüft werden - über einen etwaigen Ausschluss vom Stimmrecht ist zu entscheiden - die Stimmen müssen unter korrekter Fragestellung abgerufen werden - die abgegebenen Stimmen sind zu erfassen und gegebenenfalls auszulegen - Fragen der Gültigkeit der Stimmabgabe sind zu klären (Form, Stellvertretung, Geschäftsfähigkeit u. dgl.) - unter Beachtung der Regeln über ein etwaiges Quorum muss das Abstimmungsergebnis mit dem für den Antrag maßgeblichen Mehrheitserfordernis verglichen werden - schließlich können noch Förmlichkeiten hinsichtlich der Beschlussfeststellung zu beachten sein (Protokollierung; Verkündung; Registereintrag).

${ }_{93}$ O. Fn. 7, S. $49 \mathrm{ff}$.

94 O. Fn. 13, S. $20 \mathrm{ff}$. 
darum gehen, die ganze Lehre von den Beschlussmängeln - sie gilt als „teilweise noch umstritten und unausgereift" ${ }^{\text {95 }}$ - von neuem aufzurollen. Vom fehlerhaften Beschluss wird hier nur insofern gehandelt, als daraus etwas für das Grundverständnis des Beschlusses als Rechtsakt zu gewinnen ist.

Es erscheint vorab wichtig, die materiell-rechtliche Konformität des konkreten Beschlusses mit den bei der Beschlussfassung bestehenden Rechtsvorschriften von der Frage abzuschichten, welche prozessualen Rechtsbehelfe gegeben sind, um die Bindungswirkung des Beschlusses zu bestreiten oder zu beseitigen. Soweit man die Erfassung des Rechtsstatus des Beschlusses, mit dem etwas rechtlich „nicht in Ordnung“ ist, von den Klagemöglichkeiten her systematisiert, bedient man sich eines für unser Privatrechtsdenken ungewohnten aktionenrechtlichen Ansatzes, da das moderne Privatrechtsdenken eine primär materiell-rechtliche Sicht bevorzugt und an die durch etwaige Rechtsverletzungen begründeten Rechtsbehelfe anschließt. ${ }^{96}$

Es gibt keinen gefestigten, allgemein anerkannten Sprachgebrauch dazu, wie man den Umstand ansprechen soll, dass die Herbeiführung des Beschlusses im Einklang mit den dafür bestehenden Rechtsvorschriften erfolgt ist oder unter deren Verletzung. Man spricht wechselnd von fehlerhaften/fehlerfreien, mangelhaften/mangelfreien, nichtigen und anfechtbaren Beschlüssen. Noack hat von einer „internen Nichtigkeit“ gesprochen und damit eben die Fälle der „gestörten“ rechtlichen Abhängigkeit des Beschlusses vom weiteren Verbandsrecht gemeint. ${ }^{97}$ Gegen die Redeweise von den Fehlern oder Mängeln des Beschlusses ist an sich nichts einzuwenden. Es geht indes doch darum, ob der Beschluss als abhängiger Rechtsakt rechtmäßig oder rechtswidrig ist: Weil die Herbeiführung des Beschlusses - als eines abhängigen Rechtsakts - durch eine Vielfalt von rechtlichen Bestimmungen normiert ist, kann der Beschluss rechtmäßig oder rechtswidrig sein. Ein Beschluss ist rechtmäßig, wenn alle die Beschlussfassung betreffenden rechtlichen Bestimmungen (normativen Vorgaben) eingehalten worden sind; ein Beschluss ist rechtswidrig, wenn dies nicht der Fall ist. ${ }^{98}$ Üblich ist der Hinweis auf das Gesetz und die Satzung (den Gesellschaftsvertrag etc.) als die Rechtsquellen, die auf die Abstimmung bezügliche Rechtsnormen enthalten, deren Verletzung den Beschluss als rechtswidrig qualifiziert. Die Bezeichnung des Beschlusses als rechtmäßig oder rechtswidrig spricht wohl am besten den Umstand an, dass der Beschluss als abhängiger Rechtsakt im Einklang mit „übergeordneten“ Normen des Verbandsrechts (und der Rechtsordnung allgemein) ergehen muss. Die Kennzeichnung eines Beschlusses als rechtswid-

${ }^{95}$ K. Schmidt (o. Fn. 11), S. 444.

96 Ähnl. K. Schmidt (o. Fn. 11), S. 446 m.w.Nachw.

${ }_{97}$ O. Fn. 23, S. 49 ff. u. öfter.

${ }^{98}$ Nicht eingegangen wird hier auf die Möglichkeit, dass die Rechtswidrigkeit durch Heilung des Beschlussmangels behoben wird. 
rig erfasst auch die Fälle, in denen man sagt, der Beschluss sei nichtig. Denn auch in diesen Fällen handelt es sich darum, dass der Beschluss nicht im Einklang mit den die Beschlussfassung regelnden Normen erfolgt ist, wobei nur die Rechtsverletzung eklatant und evident ist. ${ }^{99}$ Es liegt auf der Hand, dass der nichtige Beschluss ein Unterfall des rechtswidrigen Beschlusses ist. Anfechtbarkeit und Nichtigkeit wiederum sind unterschiedliche, vornehmlich vom Prozess her betrachtete Folgen des Umstandes, dass ein Beschluss rechtswidrig ist; die Rechtswidrigkeit des Beschlusses ist stets der materiellrechtliche „Primärbefund“. Es erscheint also am ungezwungensten, den Beschluss einfach mit den Kategorien der Rechtmäßigkeit oder Rechtswidrigkeit zu erfassen, wobei sich die Nichtigkeit als eine qualifizierte Rechtswidrigkeit darstellt. Mit diesen Kategorien kann man rechtsformübergreifend arbeiten. Nun sind weitere Klassifikationen der Rechtswidrigkeit denkbar, etwa nach der Art der verletzten Rechtsnorm, nach deren Stellung in der Normhierarchie und dergleichen mehr. In diese Richtung soll hier aber nicht weitergedacht werden.

Der Umstand, dass man jeden Beschluss als entweder rechtmäßig oder rechtswidrig qualifizieren kann, stellt in Wirklichkeit eine sehr aufschlussreiche Eigenschaft des Beschlusses als Aktstyp dar. Dadurch, dass der Beschluss als rechtmäßig oder rechtswidrig qualifiziert werden kann, unterscheidet er sich vom gewöhnlichen Rechtsgeschäft. Das Rechtsgeschäft des Privatrechts ist im Allgemeinen entweder gültig oder ungültig, es entfaltet dementsprechend seine Rechtswirkungen oder es bleibt wirkungslos. ${ }^{100}$ Die Ungültigkeit des Rechtsgeschäfts ist „self-executing“. Hingegen kann man bei dem gewöhnlichen Rechtsgeschäft von Rechtmäßigkeit oder Rechtswidrigkeit eigentlich nicht sprechen. Auch im Fall eines Verstoßes gegen ein gesetzliches Verbot ( $\mathbb{1 3 4}$ BGB) handelt es sich für das Privatrecht darum, ob das Rechtsgeschäft gültig oder ungültig ist. Es sei wiederholt: „Rechtswidrigkeit“ und „Rechtmäßigkeit“ sind Attribute des Beschlusses zunächst einmal gerade im Hinblick auf den Umstand, dass der Beschluss einen abhängigen Rechtsakt darstellt, für dessen Rechtsstatus es darauf ankommt, ob er in Übereinstimmung mit den die Beschlussfassung regelnden Rechtsgrundlagen gefasst wurde oder nicht.

Eine erste Ursache für die notorische Komplexität der Beschlussmängellehre liegt in Folgendem: Für den Beschluss ist einerseits die Frage zu stellen, ob die speziellen, für die Beschlussfassung bestehenden Rechtsnormen eingehalten worden sind; insoweit geht es um die Eigenheit des Beschlusses als

99 Anders verhält es sich allenfalls bei der umstrittenen Kategorie der sogenannten Nicht-Beschlüsse, bei denen schon auf erste Sicht nicht einmal der Schein einer regulären Beschlussfassung vorliegt; dazu etwa U. Noack (o. Fn. 23), 11 f.; K. Schmidt (o. Fn. 11), S. 441 .

100 Vgl. Flume (o. Fn. 84), S. 556. 
eines abhängigen Rechtsakts. Andererseits kann der Beschluss auch einen Rechtsverstoß beinhalten, der ebenso bei einem unabhängigen Rechtsgeschäft vorkommen kann, etwa einen Verstoß gegen ein Verbotsgesetz ( $\$ 134$ BGB). Man könnte nun daran denken, diejenige „Fehlerhaftigkeit" des Beschlusses, die darin besteht, dass seine Fassung gegen das Verbandsrecht verstößt, von den Ungültigkeitsgründen abzuschichten, die ein abhängiges genauso wie ein unabhängiges Rechtsgeschäft betreffen können. ${ }^{101}$ Damit würde sich das eigentliche Beschlussmängelrecht auf die Fälle einer sub specie verbandsrechtlicher Normen „inkorrekten“ Herbeiführung des Beschlusses beschränken. ${ }^{102}$ Oder soll man das Erfordernis einer rechtlich einwandfreien Beschlussfassung über das eigentliche Verbandsrecht hinaus auch auf die von der Rechtsordnung im Übrigen aufgestellten Normen erstrecken, so dass ein inhaltlicher Verstoß des Beschlusses etwa gegen ein Verbotsgesetz ebenso wie eine sub specie verbandsrechtlicher Normen ,inkorrekte“ Herbeiführung des Beschlusses behandelt wird (so wohl die h.L.)? Man kann die Frage auch so stellen: In welchem Umfang sollen hinsichtlich des Beschlusses Rechtsverstöße, die beim gewöhnlichen Rechtsgeschäft „self-executing“ sind, indem sie ipso iure dessen Gültigkeit verhindern, dem ganz anders gearteten Mechanismus unterstellt werden, mit dem zunächst einmal die Fälle erfasst werden sollen, in denen die Beschlussfassung nicht im Einklang mit den für sie bestehenden verbandsrechtlichen Bestimmungen erfolgt ist? An dieser Stelle soll nicht versucht werden, an der Beantwortung dieser Problemstellung weiterzuarbeiten.

\section{Rechtswidrigkeit des Beschlusses und Beschlussgeltung}

Die inhaltliche Bindungswirkung des rechtswidrigen Beschlusses kann unumwunden als das Zentralproblem der Beschlusslehre bezeichnet werden. Dieses Problem hat in der Rechtsgeschäftslehre ansonsten kein vergleichbares Gegenstück, es ist insofern elementarer Natur. Im Grundsatz kann man es nicht anders halten, als dass man auch dem rechtswidrigen Beschluss Bindungswirkung zuerkennt. Die Zuständigkeitsordnung des Verbandes wäre unterlaufen, wenn andere Organe und jedes einzelne Mitglied oder gar Verbandsfremde das Recht hätten, sich über festgestellte Beschlüsse hinwegzusetzen, indem sie deren Rechtmäßigkeit bestreiten. Dass die Versammlung für sich konstatiert hat, so und so habe man beschlossen, bleibt auch dann eine Respekt verlangende Setzung, wenn der Beschluss rechtswidrig gefasst

101 Man muss bezweifeln, ob die hier mögliche Differenzierung sich mit der Unterscheidung zwischen „formellen“ und „materiellen“ Mängeln deckt; hierfür indes K. Schmidt (o. Fn. 11), S. 440.

${ }^{102}$ Für eine Abschichtung in diesem Sinne hat sich namentlich Noack in dem in Fn. 23 zit. Werk eingesetzt. 
worden sein sollte. Der rechtswidrige Beschluss - die nichtigen Beschlüsse seien hier einmal ausgeklammert - ist also kein bloßer Schein-Beschluss, dessen Nicht-Geltung allein schon durch Nachweis des „Beschlussmangels“ aufgedeckt werden könnte. Vielmehr ist auch der rechtswidrige Beschluss ein zunächst gültiger, verbindlicher Beschluss.

Es ist mithin nicht erst eine etwaige gesetzliche Regelung über die Anfechtbarkeit der (Hauptversammlungs-)Beschlüsse, die dem Beschluss eine von seiner Rechtmäßigkeit unabhängige Bindungswirkung verleiht, sondern es ist der Sinn des Endaktes, der die Beschlussfassung abschließt, für das Beschlussverfahren ein eindeutiges, abschließendes Ergebnis mit verbindlicher Wirkung für den Verband und seine Mitglieder festzustellen. Die Möglichkeiten des gerichtlichen Angriffs, die von Verband zu Verband unterschiedlich sind, versuchen, dieser Bindungswirkung zu entsprechen, die der Beschluss als solcher rechtsformunabhängig entfaltet. Die bekannten Einschränkungen des gerichtlichen Angriffs gegen den Beschluss sind auf dessen genuine Bindungswirkung hin angelegt; diese Einschränkungen sind aber nicht der Grund für die Bindungswirkung.

Die Eigenschaft des Beschlusses, Bindungswirkung zu entfalten grundsätzlich ohne Rücksicht auf eine etwaige Rechtswidrigkeit, wohnt der einzelnen Stimmabgabe nicht inne; diese ist gültig oder ungültig. Wäre der Beschluss wirklich nicht mehr als die Vereinigung der gleichgerichteten Stimmabgaben, wäre es ein schwer erklärlicher Fall von Emergenz, dass dem Beschluss eine Rechtsqualität zukommt, die die einzelnen Stimmabgaben noch nicht aufweisen sollten.

\section{Die Beseitigung der Beschlussgeltung durch Richterspruch}

Die Bindungswirkung, die der Beschluss für den Verband und auch dem einzelnen Verbandsmitglied gegenüber entfaltet, wäre infrage gestellt, wenn jeder Einzelne die Rechtmäßigkeit des Beschlusses bestreiten und den Verband in eine bilaterale Auseinandersetzung um die Rechtmäßigkeit des Beschlusses verwickeln könnte. Wohl kann die Versammlung ihren Beschluss aufheben oder einen Gegenbeschluss fassen. ${ }^{103}$ Es wäre aber mit der Zuständigkeitsordnung des Verbandes unvereinbar, wenn andere Verbandsorgane oder einzelne Mitglieder sich aufgrund ihrer Rechtsansicht von der Bindungswirkung des Beschlusses einseitig freisprechen könnten. Für die Beseitigung der Bindungswirkung des Beschlusses kann nur eine Instanz außerhalb des Verbandes zuständig sein. Die Beseitigung der Bindungswirkung des Beschlusses (von regelrechten Nicht-Beschlüssen sei hier einmal abge-

${ }^{103}$ Zum Unterschied s. Ernst (o. Fn. 48), Rn. 276, 279. 
sehen) wegen rechtlicher Beanstandung kann nur durch Richterspruch erfolgen. Ein Urteil, das die Rechtswirksamkeit des Beschlusses negiert, sollte insoweit immer auch Gestaltungswirkung erga omnes haben, auch dann, wenn es auf eine allgemeine Feststellungsklage hin ergeht. ${ }^{104}$ Wenn ein Mitglied auf Zahlung rückständiger Beiträge verklagt ist, die es sich zu zahlen weigert, weil es den Beschluss über die Beitragserhebung nicht für rechtmäßig zustande gekommen hält, setzt die Abweisung der Klage voraus, dass der Richter die Bindung an den Beschluss negiert. Auf das prozessuale Problem, ob es sich um eine Vorfrage handelt oder ob ein Zwischenfeststellungsurteil hinsichtlich der Ungültigkeit des Beschlusses zu beantragen wäre, soll hier nicht weiter eingegangen werden.

Eine oft übersehene Folge der richterlichen Beschlussaufhebung besteht darin, dass die Versammlung über den Antrag, über den die Abstimmung stattgefunden hatte, nicht entschieden hat. Da mit dem Antragsrecht das Recht verbunden ist, dass über den Antrag ordnungsgemäß entschieden wird, muss über den Antrag neu abgestimmt werden. Gerade aus diesem Grund besteht ein Rechtsschutzinteresse an der Ungültigerklärung eines Negativbeschlusses.

Der Umstand, dass die Rechtswidrigkeit des Beschlusses - von Fällen der Nichtigkeit einmal abgesehen - nur mittels gestaltenden Urteils zu einer Aufhebung der Bindungswirkung des Beschlusses führt, stellt eine ganz andere „Mechanik“ dar als die der Gültigkeit/Ungültigkeit von Rechtsgeschäften. Das ungültige (unwirksame) Rechtsgeschäft zeitigt einfach nicht die intendierte Rechtswirkung. Wie bereits erwähnt, ist die Ungültigkeit des Rechtsgeschäfts im Allgemeinen „self-executing“. ${ }^{105}$ Wird das ungültige Rechtsgeschäft irrig für wirksam gehalten, so ist die ihm beigelegte Wirksamkeit nur eine scheinbare. Stellt sich die Unwirksamkeit erst im Zuge eines Rechtsstreits heraus, muss der Richter lediglich erkennen, wie sich die Rechtslage aufgrund des Ausbleibens der intendierten Rechtsfolgen darstellt. Demgegenüber handelt es sich bei der Anfechtung von Beschlüssen um eine richterliche Gestaltung der Rechtslage aufgrund einer repressiven Rechtmäßigkeitskontrolle. ${ }^{106}$ Während die Ungültigkeit eines Rechtsgeschäfts, weil „per se" eintretend, ohne weiteres jedermann betrifft, für den es auf Wirkun-

${ }^{104}$ Gestaltungsurteile sind nach Schlosser solche Urteile, die eine Rechtsfolge verfügen, „auf die sich ohne Erlass und meist auch ohne formelle Rechtskraft des Urteils, das die Rechtsfolge beinhaltet, niemand berufen kann"; P. Schlosser, Gestaltungsklagen und Gestaltungsurteile, 1966, S. 37; ähnl. M. Sogo, Gestaltungsklagen und Gestaltungsurteile des materiellen Rechts und ihre Auswirkungen auf das Verfahren, 2007, S. 10.

105 S. Flume (o. Fn. 84), S. 556.

106 Für diese Kontrolle kann man die Frage des Kontrollgegenstandes, des Kontrollmaßstabs und auch die der Kontrolldichte stellen; s. auch L. Fastrich, Funktionales Rechtsdenken am Beispiel des Gesellschaftsrechts, 2001, S. 24 ff. 
gen des Rechtsgeschäfts ankommt, stellt sich für die prozessuale Anfechtung des Beschlusses das Problem der Klagebefugnis, auf das hier indes nicht weiter eingegangen werden soll.

Die richterliche Überprüfung des angefochtenen Beschlusses muss dessen objektive Rechtswidrigkeit ergeben, damit dieser aufgehoben werden kann. Der Richter muss zu einem eigenständigen Urteil dazu kommen, ob der Beschluss rechtmäßig oder rechtswidrig ist. Wenn der Richter zu dem Ergebnis kommt, es wäre derselbe Beschlussinhalt festzustellen gewesen, wie er in der Versammlung festgestellt wurde, unterbleibt die Aufhebung des Beschlusses. Manche pflegen dies so auszudrücken, dass die Rechtsverletzung für den angefochtenen Beschluss ursächlich geworden sein muss. ${ }^{107}$ Die Einzelheiten sind bekanntlich sehr umstritten; es genügt, die Stichworte potenzielle Kausalität und Relevanztheorie zu nennen. ${ }^{108}$ Der kernhafte Sachgrund für diese Handhabung der Beschlusskontrolle liegt darin, dass auch der rechtswidrige Beschluss eine $\mathrm{zu}$ beachtende Konstitutivwirkung entfaltet, die ihm nicht genommen werden muss, wenn bei Beachtung aller normativen Bestimmungen ein Beschluss desselben Inhalts gefasst worden wäre.

Wieder sei der Befund benutzt, um an ihm die Vorstellung zu überprüfen, der Beschluss entstehe mit der Vereinigung von Willenserklärungen zu einem mehrseitigen Rechtsgeschäft. Wäre der Beschluss wirklich ein Rechtsgeschäft stricto sensu, wäre für ihn die Frage, ob er als nur scheinbarer Beschluss ungültig ist, genauso zu stellen wie für das gewöhnliche Rechtsgeschäft. Für die Rechtsgeschäftslehre ist insbesondere die Vorstellung ungewöhnlich, man könne bei Gültigkeitsproblemen zu einer hypothetischen Betrachtung übergehen und die Geltung eines Rechtsgeschäfts darauf stützen, dass es zu dessen Abschluss hätte kommen müssen. Wie schwer sich die allgemeine Rechtsgeschäftslehre mit solchen Überlegungen tut, zeigt sich an der Diskussion des Falles, dass ein Anfechtungsgegner den Anfechtenden an einer hypothetischen, weil nicht real abgegebenen Erklärung desjenigen Inhalts festhalten will, der dem Irrenden tatsächlich vorgeschwebt hatte. ${ }^{109}$

\section{Die gerichtliche Durchsetzung des Anspruchs auf Beschlussfeststellung}

Nehmen wir zum Beispiel an, dass die JA- und NEIN-Stimmen nicht korrekt gezählt worden sind. Es wird festgestellt, der Antrag sei abgelehnt; bei richtiger Zählung hätte die Annahme des Antrags festgestellt werden müssen.

${ }^{107}$ K. Schmidt (o. Fn. 11), S. 443 bei u. mit Fn. 50.

108 S. für die GmbH Zöllner, in: Baumbach/Hueck, GmbHG, 19. Aufl. 2010, $\ 47$ Rn. 60 ff.; für den Verein: Reuter, in: MünchKomm-BGB, 6. Aufl. 2012, \32 Rn. 55.

109 S. Leenen (o. Fn. 16), S. 292 f.; T. Lobinger, AcP 195 (1995), 274 ff. 
Hier ist der festgestellte Beschlussinhalt (Ablehnung) gleichwohl „der“ gefasste Beschluss, dem im Verband Bindungswirkung zukommt; er ist - handelt es sich um eine Hauptversammlung - anerkanntermaßen der Gegenstand der aktienrechtlichen Anfechtungsklage. ${ }^{110}$ Derjenige (gedachte) Beschluss, der bei korrekter Auszählung festzustellen gewesen wäre, ist nach der hier vertretenen Ansicht nicht der „wirkliche“, „eigentliche“ Beschluss der Versammlung. Dieser hypothetische Beschluss, der bei richtigem Vorgehen hätte festgestellt werden müssen, existiert nicht; man kann nur sagen, dass ihm bei rechtmäßigem Vorgehen zur Existenz hätte verholfen werden müssen. Dies bedarf der Hervorhebung, weil nach der Vorstellung, der Beschluss sei einfach die Vereinigung der ihn befürwortenden Stimmabgaben, dieser Beschluss tatsächlich gefasst, aber bloß noch nicht verkündet worden ist. Vom hier vertretenen Standpunkt aus kommt in der Situation, dass die festgehaltenen Umstände und Ergebnisse der Abstimmung die zweifelsfreie Folgerung zulassen, es hätte in der Versammlung ein Beschluss eines bestimmten Inhalts festgestellt werden müssen, eine Leistungsklage auf Beschlussfeststellung in Betracht. Lehrbeispiel ist die Feststellung des Negativbeschlusses aufgrund der irrigen Annahme, der Antrag erfordere zu seiner Annahme eine qualifizierte Mehrheit; die lediglich erforderliche einfache Mehrheit war erreicht worden; als Beschluss wäre die Annahme des Antrags festzustellen gewesen.

Die Klage gründet sich materiell-rechtlich auf den Anspruch des Stimmberechtigten, Beschlussfeststellung verlangen zu können; dieser Anspruch ist Ausfluss des Stimmrechts. ${ }^{111}$ Aktivlegitimiert ist jeder, der an der fraglichen Abstimmung teilgenommen hat; nur der Mitstimmende kann ja für seine Stimmabgabe den Anspruch geltend machen, dass diese in einer richtigen Beschlussfeststellung berücksichtigt wird. ${ }^{112}$ Passivlegitimiert ist der Verband. Der Klageantrag richtet sich darauf, den Verein (die Aktiengesellschaft usw.) zu verpflichten, den Beschluss so und so festzustellen. Für die Vollstreckung kann $\ 894 \mathrm{ZPO}$ analog angewendet werden. Die verurteilungsgemäß vorgenommene Beschlussfeststellung wirkt schon als solche erga omnes; ebenso muss es sich verhalten, wenn die verurteilungsgemässe Beschlussfeststellung analog $\$ 894 \mathrm{ZPO}$ fingiert wird. Die Klage, mit welcher der Verband verpflichtet werden soll, einen bestimmten Beschlussinhalt festzustellen, kann mit einer Klage, mit welcher dem festgestellten Beschluss die Geltung genommen wird, verbunden werden. Die aktienrechtliche Praxis lässt be-

110 BGHZ 104, 66; s. auch BGH ZIP 2002, 1684.

111 Siehe oben VI. a.E.

112 Da der „fehlerhafte“ Beschluss nicht nur die Mitstimmenden bindet, ist die Aktivlegitimation für die Anfechtungsklage weiter zu fassen, gelten insoweit im Grundsatz alle Verbandsangehörigen als klagebefugt. 
kanntlich die Verbindung der bloß kassatorisch wirkenden ${ }^{113}$ Anfechtungsklage mit einer sog. positiven Beschlussfeststellungsklage zu. ${ }^{114} \mathrm{Um}$ eine Feststellungsklage i.S.d. $\$ 256$ ZPO handelt es sich m.E. - entgegen der allgemeinen Ansicht in Lehre und Rechtsprechung - indes nicht, weil der „richtige" Beschluss mangels Beschlussfeststellung nicht gefasst ist. Sofern anhand des Prozessstoffes der Beschlussinhalt, wie er richtig hätte festgestellt werden müssen, zweifelsfrei bestimmt werden kann, spricht das Gericht eine Verpflichtung des Verbandes aus, den Beschluss genau dieses Inhalts festzustellen. Wegen $\ 894 \mathrm{ZPO}$ ist der Unterschied zu einem Feststellungsurteil geringfügig. Gelegentlich wird für die richtige Beschlussfeststellung ein erneutes Tätigwerden des Verbandes unumgänglich sein (z.B. Neuauszählung); in diesem Fall ist der Verband durch Urteil zu verpflichten, den Beschluss unter Beachtung der Rechtsauffassung des Gerichts von neuem festzustellen. Man könnte von Verpflichtung- und Bescheidungsurteilen sprechen.

\section{Gültigkeit und Ungültigkeit der Stimmabgabe im Verhältnis zu Rechtmäßigkeit und Rechtswidrigkeit des Beschlusses}

Wir wenden uns noch einem wohlbekannten Problem zu, wieder nur zu dem begrenzten Zweck, das Verhältnis von Stimmabgabe und Beschlussfassung zu erhellen. Es geht um das Zusammenspiel von Gültigkeit/Ungültigkeit der Stimmabgabe und Rechtmäßigkeit/Rechtswidrigkeit des Beschlusses. Für die einzelne Stimmabgabe kann man von Gültigkeit und Ungültigkeit sprechen. ${ }^{115}$ Es ist die Frage, wie sich die Ungültigkeit einer für gültig erachteten Stimmabgabe auf den Beschluss auswirkt. Als Beispiel kann der Fall genommen werden, dass ein Stimmrechtsausschluss unbeachtet geblieben ist. Geht man aus von der Sicht des Beschlusses als einem aus den einzelnen Stimmabgaben zusammengesetzten Rechtsakt, müsste die Ungültigkeit der einzelnen Stimmabgabe die Gültigkeit des Beschlusses als des Gesamtakts infrage stellen: Beim mehrseitigen Vertrag führt die Ungültigkeit einer der Vertragserklärungen zur Unwirksamkeit des Vertrags. Beim Beschluss müsste man fragen, ob bei Abzug der zu Unrecht für gültig gehaltenen Stimme die übrigen Stimmen noch für einen Mehrheitsbeschluss ausreichen; ist dies der Fall, so müsste die Ungültigkeit der einzelnen Stimmabgabe folgenlos bleiben - der Beschluss wäre auch gar nicht rechtswidrig -; war der Beschluss dagegen mit einer Mehrheit von nur einer Stimme zustande gekommen, dürfte

113 Statt aller Roth, in: Roth/Altmeppen, GmbHG, 7. Aufl. 2012, $\mathbb{S} 47$ Rn. 155 f.; K. Schmidt (o. Fn. 11), S. 438 f., 441 f., jew. m.w.N.

${ }^{114}$ Roth, in: Roth/Altmeppen, GmbHG, 7. Aufl. 2012, $\$ 47$ Rn. 131.

115 Siehe oben VII. 
der Beschluss, wenn sich die Ungültigkeit einer Stimme herausstellt, ipso iure nicht zustande gekommen sein. So verfährt man beim Beschluss indes nicht. ${ }^{116}$ Die Ungültigkeit einer für gültig gehaltenen Stimmabgabe nimmt als solche dem Beschluss nicht die Geltung; wohl aber kann der Beschluss - weil gültig, aber rechtswidrig - durch prozessuale Anfechtung zu Fall gebracht werden. Es verhält sich so, dass sich der Sachverbalt, aufgrund dessen der Beschluss festgestellt wurde, anders darstellt als (irrig) angenommen, indem eine Stimme als gültig angenommen wurde, die in Wirklichkeit nicht gültig abgegeben war. Bei der prozessualen Anfechtung des Beschlusses wegen der Ungültigkeit einzelner Stimmabgaben stellt sich typischerweise die schon angesprochene Frage, ob von einer Aufhebung des (rechtswidrigen) Beschlusses abgesehen werden kann, weil bei richtiger Beurteilung der Gültigkeit einer konkreten Stimmabgabe der Beschluss gleichen Inhalts zu fassen gewesen wäre. ${ }^{117}$ Wenn die Stimme, die von Anfang hätte ausgeschieden werden müssen, nicht entscheidungserheblich war, unterbleibt die gerichtliche Beschlussaufhebung. Dies ändert aber nichts daran, dass ein Rechtsverstoß stattgefunden hat; dieser bietet nur keine hinreichende Grundlage für die Beseitigung des Beschlusses.

Für das Thema dieser Untersuchung liegt der wesentliche Befund hier darin, dass die Ungültigkeit einer Stimmabgabe und der Beschlussmangel raumbildlich gesprochen - auf zwei verschiedenen Ebenen liegen, so dass die Ungültigkeit der Stimmabgabe, wenn sie den Beschluss zu Fall bringen soll, in einen Beschlussmangel gleichsam transponiert werden muss. Auch in der Frage der „Mängel“ spiegelt sich damit der Befund, dass das Verhältnis, wie es zwischen Stimmabgabe und Beschluss besteht, nicht das Verhältnis des Teils zum Ganzen ist.

\section{Anfechtbare Stimmabgaben}

Sehen wir insbesondere auf den Fall, dass eine Stimmabgabe ihre Gültigkeit durch eine Anfechtung verliert. Die Anfechtung der Stimmabgabe entsprechend $\$ \mathbb{S} 119 \mathrm{ff}$. BGB ist von der zivilprozessualen Beschlussanfechtung zu unterscheiden. Die Anfechtung der Stimmabgabe nach $\mathbb{S} 119 \mathrm{ff}$. BGB erfolgt durch Anfechtungserklärung gegenüber dem Versammlungsleiter, nach Schluss der Versammlung aber gegenüber dem Verband; die prozessuale Anfechtung des Beschlusses richtet sich stets gegen den Verband. Wesentlich ist dabei, dass für die bürgerlich-rechtliche Anfechtung einer Stimmabgabe 
etwa wegen Täuschung $(\mathbb{1 2 3} \mathrm{BGB})^{118}$ das Anfechtungsobjekt die Stimmabgabe als solche ist und nicht auch der Beschluss, da die Stimmabgabe nicht einfach ein Teil des Beschlusses ist. Den Beschluss kann der Stimmende schon deswegen nicht nach $\mathbb{\$} \$ 119 \mathrm{ff}$. BGB anfechten, weil er nicht dessen auctor ist; auctor des Beschlusses ist vielmehr die Versammlung als ganze. Es verhält sich damit wesentlich anders als beim Vertragsschluss, bei dem mit der Anfechtung einer der beiden Vertragserklärungen zugleich der Vertrag zu Fall gebracht wird; man kann daher beim Vertrag sagen, dass sich die Anfechtung, obwohl das Anfechtungsobjekt eine der Vertragserklärungen ist, doch unmittelbar gegen die Vertragsgeltung richtet. ${ }^{119}$ Wenn durch die Irrtumsanfechtung der einzelnen Stimmabgabe auch der Beschluss zu Fall gebracht werden soll, muss zusätzlich zur Abgabe der bürgerlich-rechtlichen Anfechtungserklärung hinsichtlich der Stimmabgabe noch prozessual um die richterliche Beseitigung des Beschlusses nachgesucht werden. Die Anfechtung der Stimmabgabe entsprechend $\mathbb{} 143$ BGB beinhaltet - selbstverständlich nicht die prozessuale Beschlussanfechtung, zu der es einer Klageerhebung bedarf. Wenn jemand zivilprozessual gegen die Beschlussgeltung mit der Berufung auf die Ungültigkeit seiner Stimmabgabe vorgeht und diese Ungültigkeit sich erst infolge einer Anfechtung der Stimmabgabe entsprechend $\mathbb{S} 142 \mathrm{ff}$. BGB ergibt, wird man in dem prozessualen Vorgehen gegen den Beschluss auch die konkludente Anfechtung der Stimmabgabe entsprechend $\mathbb{S} 142 \mathrm{ff}$. BGB sehen können. Zur prozessualen Anfechtung des Beschlusses, bei dessen Fassung eine Stimme abgegeben wurde, die wirksam angefochten worden ist, ist übrigens nicht nur derjenige aktivlegitimiert, der seine Stimmabgabe angefochten hat. Dagegen ist die bürgerlich-rechtliche „Vernichtung“" der Stimmabgabe, die von einem Willensmangel begleitet war, als Individualbefugnis dem betroffenen Stimmberechtigten vorbehalten. ${ }^{20}$

Wer seine Stimmabgabe wegen eines Erklärungs- oder Inhaltsirrtums nach $\$ 119$ BGB anficht, begründet für den Verband einen Anspruch auf den Ersatz des Vertrauensschadens nach $\ 122$ BGB. Kommt es in der Folge der bürgerlich-rechtlichen Anfechtung zu einer richterlichen Beseitigung des Beschlusses und wird damit eine erneute Abstimmung erforderlich, ${ }^{121}$ muss der Anfechtende für die Kosten aufkommen.

118 Bei der Anfechtung wegen arglistiger Täuschung, die von einem Dritten ausgeht, wird wegen $\ 123$ II BGB verlangt, dass der Versammlungsleiter die Täuschung kannte oder kennen musste; ausreichend muss auch sein, dass ein Organ, das den Verband vertritt, die Dritttäuschung kannte oder kennen musste, vgl. Flume (o. Fn. 14), S. 250; s. aber auch noch die folgende Fn.

119 Vgl. zu dieser Frage auch Leenen (o. Fn. 16), S. 102; ders., Jura 1991, 393.

120 Sofern Stimmberechtigte getäuscht worden sind, ist zu erwägen, die arglistige Verfälschung der Abstimmung als Nichtigkeitsgrund anzusehen.

121 Oben XII. 
Interessant ist noch, ob die - nur kassatorisch wirkende - bürgerlichrechtliche Anfechtung einer Stimmabgabe entsprechend $\$ \mathbb{S} 119 \mathrm{ff}$. BGB nicht bloß die prozessuale Anfechtung und Beseitigung des Beschlusses eröffnet, sondern auch zur Grundlage einer Klage auf positive Beschlussfeststellung gemacht werden kann. ${ }^{122}$ Anstelle der durch Anfechtung entsprechend S 119 ff. BGB weggefallenen Stimme kann eine Stimmabgabe anderen Inhalts nicht fingiert werden; auch der Anfechtende kann nicht eine neue Stimmabgabe „nachschieben“, selbst wenn er geltend macht, diese hätte er ohne den Willensmangel so abgegeben. Allerdings kann der bloße Fortfall der ungültig gewordenen Stimme ein anderes Beschlussergebnis bedingen, wenn es für den Beschlussinhalt auf jede JA- bzw. NEIN-Stimme angekommen war. Beispiel: Ein Antrag war bei Stimmengleichheit als abgelehnt festgestellt worden. Einer der Mitstimmenden, der eine NEIN-Stimme abgegeben hatte, ficht seine Stimmabgabe nach $\ 123$ BGB wegen Täuschung an. Er kann dem ablehnenden Beschluss mit einer Feststellungsklage nach $\mathbb{} \$ 256$ ZPO oder - soweit es um Aktiengesellschaft oder GmbH geht - mit einer Anfechtungsklage nach $\$ 246$ AktG die Geltung nehmen lassen. Kann damit die Leistungsklage darauf verbunden werden, dass der Verband den Antrag als angenommen festzustellen hat oder muss es dabei bleiben, dass die Abstimmung über den - nicht beschiedenen - Antrag wiederholt werden muss? ${ }^{123}$ Die Neuabstimmung erscheint als die richtige Lösung.

\section{Zur Rechtsnatur des Beschlusses}

Von dem hier vertretenen Standpunkt aus, demzufolge sich der Beschluss gerade nicht aus den einzelnen Stimmabgaben zusammensetzt, müssen die Fragen nach der Rechtsnatur der Stimmabgabe und nach der Rechtsnatur des Beschlusses getrennt beantwortet werden. ${ }^{124}$ Zur Rechtsnatur der Stimmabgabe wurde bereits Stellung genommen. ${ }^{125}$

Fassen wir zunächst zusammen, was über den Beschluss rechtlich ausgesagt werden kann. Der Beschluss ist ein einseitiger Rechtsakt, der von der Versammlung als ganzer herrührt, für die er (typischerweise) vom Versammlungsleiter festgestellt wird. Diese Inhaltsfeststellung ist für den Beschluss konstitutiv. Die Versammlung als ganze ist der Urheber des Beschlusses. Der Beschluss ist nicht ein mehrseitiges Rechtsgeschäft zwischen den Zustimmenden, an welches die Nichtzustimmenden als extranei wegen des Mehrheitsprinzips gebunden wären. Der Beschluss ist vielmehr ein einziger und

122 Oben XIII.

123 Vgl. oben XII.

${ }^{124}$ Ebenso K. Schmidt (o. Fn. 11), S. 437.

125 Oben VII. 
einheitlicher Akt der Setzung, der den Verband, seine Organe und seine Mitglieder gleichermaßen bindet, die Mitglieder ohne Rücksicht darauf, ob und wie sie in der zugrunde liegenden Abstimmung gestimmt haben. Dieses Konzept schließt bruchlos den einstimmigen Beschluss und den Beschluss durch den Alleingesellschafter ein, ebenso den Negativbeschluss.

Der Beschluss ist ein abhängiger Rechtsakt, der nur auf der Grundlage einer verbandsrechtlichen Zuständigkeits- und Verfahrensordnung getätigt werden kann. Insbesondere bedarf es für die Setzung des Beschlusses einer Zuständigkeit, die bei dem Verbandsorgan als ganzer liegt. Es ist eine Besonderheit des Beschlusses als Konstitutivakt, dass er, weil er auf die verbandsrechtliche Zuständigkeits- und Verfahrensordnung bezogen, also abhängiger Natur ist, rechtmäßig oder rechtswidrig sein kann. Eine etwaige Rechtswidrigkeit steht aber der Geltung des Beschlusses nicht ohne weiteres entgegen; hierin liegt ein wesentlicher Unterschied zum Rechtsgeschäft stricto sensu.

Der Beschluss erlangt erst und nur durch seine Feststellung Geltung. Diese ist konstitutiver Bestandteil der Beschlussfassung, stellt deren Endakt dar. Die Beschlussfeststellung schließt einen Rechtsanwendungsakt ein. Eine regelrechte Empfangszuständigkeit gibt es für die Beschlussfeststellung im Allgemeinen nicht. Eine bestimmte Form (z.B. Protokollierung, Registereintrag) ist nur einzuhalten, wo sie durch Gesetz oder Satzung vorgeschrieben ist; auch ohne Formerfordernis muss man für die Beschlussfeststellung aus der Natur der Sache heraus eine ausdrückliche Erklärung verlangen.

Man spricht von dem Beschluss auch als der Bekundung eines sogenannten „Gesamtwillens“. Diesem „Gesamtwillen“ entspricht jedoch keine psychische Tatsache. Es fehlt schon an einem menschlichen Subjekt, bei dem dieser „Gesamtwille“ als echte innere Tatsache existieren könnte. ${ }^{126}$ Nur die Stimmenden als Organwalter haben einen psychischen Willen, nicht aber die Versammlung als Verbandsorgan. Das, was durch den Beschluss der Versammlung als Wille des Verbandes gilt, gilt aber auch gar nicht aufgrund dessen, dass es von irgendeiner natürlichen Person oder von einer Mehrheit von Personen im Sinne einer psychologischen Tatsache "gewollt" wird. ${ }^{127}$ Freilich: in der einzelnen Stimmabgabe drückt sich ein "Wollen“ aus, das als psychologische Tatsache beim Stimmenden gegeben ist. Der Beschluss als Rechtsakt ist dagegen das rechtlich konstituierte Ergebnis eines Verfahrens, nach dessen gelungenem Abschluss ein bestimmter Beschlussinhalt der Versammlung (und über diese

${ }^{126}$ Mit Recht kritisch gegenüber der Redeweise vom „Gemeinschaftswillen“, „kollektivem Willen“ u. dergl. bereits Baltzer (o. Fn. 7), S. $174 \mathrm{f}$.

127 Es gibt Abstimmungsverfahren, bei welchen das Beschlussergebnis nur von einer Minderheit der Beteiligten im Sinne einer psychologischen Tatsache „gewollt" worden ist; s. z.B. das Abstimmungsverfahren nach $\$ 196$ II GVG zur Ermittlung des vom richterlichen Spruchkörper mehrheitlich bevorzugten Betrages; s. dazu W. Ernst, JZ 2012, Heft 13. 
dem Verband) zugerechnet wird. Auch bei der Beschlussfassung durch eine einzelne Person wird deren „Wollen“, wie es sich in ihrer Stimmerklärung ausdrückt, in den - hier inhaltsgleichen - Beschluss als eine der Versammlung (und über diese dem Verband) zugerechnete Entscheidung transponiert.

Weil der Beschluss nicht irgendeinem realen psychischen Willen Ausdruck gibt, handelt es sich nicht um eine Willenserklärung. ${ }^{128}$ Die Vorschriften, die das BGB für die Willenserklärung aufstellt, beziehen sich auf die Erklärung eines psychischen Wollens, das als psychologische, innere Tatsache der Wirklichkeitswelt angehört; hieran fehlt es beim Beschluss. Die Vorschriften, die das BGB für die Willenserklärung aufstellt, sind daher auf den Beschluss weder direkt noch analog anwendbar. So wäre es ganz unpassend, entsprechend $\int \mathbb{S} 119 \mathrm{ff}$. BGB eine Anfechtung der Beschlussfeststellung zuzulassen (im Unterschied zur Anfechtung einer einzelnen Stimmabgabe durch den Stimmenden). Man könnte schon nicht angeben, wer die Person ist, der ein Irrtum unterlaufen ist. Auch die $\mathbb{S}$ 116-118 BGB sind nicht anwendbar. Gegenüber der Beschlussfeststellung als einem Akt der Setzung ist jedoch die Möglichkeit der Berichtigung gegeben, ${ }^{129}$ die man entsprechend $\int \mathbb{S} 319 \mathrm{f}$. ZPO oder $\ 42 \mathrm{VwVfG}$ handhaben kann. Fragen der Geschäftsfähigkeit stellen sich im Allgemeinen nicht. ${ }^{130}$ Soweit für den Beschluss ein Formerfordernis besteht, wie z.B. nach $\ 130 \mathrm{I} 1 \mathrm{AktG}$, kann $\$ 125 \mathrm{BGB}$ angewendet werden; ${ }^{131}$ hier bezieht sich die Form, wie bereits angesprochen, nur auf die Beschlussfeststellung und nicht auf die einzelnen Stimmabgaben.

Vergleichen wir nun die Stimmabgaben als einseitige Erklärungen, die man in gewissem Umfang wie Willenserklärungen behandeln kann und die man auch als solche bezeichnen mag, mit dem Konstitutivakt der Beschlussfeststellung, so sind die Stimmabgaben und die Beschlussfeststellung von wesentlich verschiedener Rechtsnatur. Es ist hier nicht so sehr die Frage, ob Stimmabgabe und Beschluss als Willenserklärung bzw. als Rechtsgeschäft zu bezeichnen sind: Vielmehr ist es der entscheidende Befund, dass die Stimmabgaben und der festgestellte Beschluss rechtlich von wesentlich unterschiedlichen Qualitäten sind, und dass deshalb die Vorstellung kaum zutreffen kann, die Stimmabgaben seien bereits die Teilakte des Beschlusses, zu dem sie sich ohne weiteres Zutun vereinigen würden.

Mit den vorstehenden Einzelheiten ist der Beschluss als Rechtsakt im Wesentlichen beschrieben. Ob man einen Rechtsakt, der sich durch die geschilderten Einzelheiten auszeichnet, als Rechtsgeschäft bezeichnet, hängt

128 Zutr. K. Schmidt (o. Fn. 11), S. 436: „Der Beschluss ist [...] nicht [...] Willenserklärung i.S. der $\$ \mathbb{S} 116$ ff. BGB.“

${ }_{129}$ Siehe K. Schmidt/Seibt, in: Scholz, GmbHG, 10. Aufl. 2007, $\$ 48$ Rn. 53 m.w.N.

${ }_{130} \mathrm{Ob}$ man eine Beschlussfeststellung, die durch einen volltrunkenen Versammlungs-

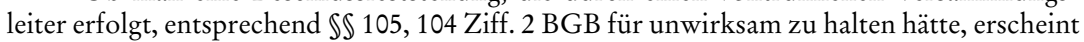
zweifelhaft.

131 Einzelheiten etwa bei Noack (o. Fn. 23), S. 28 ff. 
von der Definition des Rechtsgeschäfts ab. Die Erfassung des Beschlusses als Rechtsakt kann durch die kategoriale Einordnung, ob man den Beschluss nun als Rechtsgeschäft bezeichnet, wohl nicht weiter gefördert werden: Der Begriff des Rechtsgeschäfts ist als Begriff der systematischen Schuljurisprudenz nicht gesetzlich festgeschrieben; er kann daher wechselnd umschrieben werden und wird je nachdem den Beschluss mitumfassen oder auch nicht. ${ }^{132}$

\section{Rück- und Ausblick}

Es sind rund 100 Jahre vergangen, seitdem von Tubr den Beschluss, in einer gewissen Differenz zu Otto von Gierke, scheinbar bruchlos in die Lehre vom Rechtsgeschäft integriert hat, indem er ihn als mehrseitiges Rechtsgeschäft definierte, das aus den einzelnen, gleichgerichteten Stimmabgaben zusammengesetzt sei. Diese Vorstellung hat sich als ausgesprochen erfolgreich erwiesen. Die vorstehenden Überlegungen wollen hingegen gezeigt haben, dass die Definition des Beschlusses als das Zusammentreffen gleichgerichteter Stimmerklärungen in einem mehrseitigen Rechtsgeschäft trotz der suggestiven Systematisierungsleistung, die mit dieser Vorstellung einhergeht, auf Schritt und Tritt nicht überzeugen kann. ${ }^{133}$ Wenn man diese Vorstellung preisgibt, wie es unausweichlich erscheint, kann der Rechtsakt, der die Beschlussfassung bewirkt, nur in der Beschlussfeststellung gesehen werden, an deren konstitutiver Bedeutung für die Beschlussfassung damit - rechtsformunabhängig - kein Weg vorbeigeht.

P.S.: Nach Drucklegung habe ich Kenntnis erhalten von J. Busche, Zur Rechtsnatur und Auslegung von Beschlüssen, Festschrift Säcker, 2011, S. 45 ff. Die Überlegungen Busches treffen sich in Vielem mit den Thesen dieser Untersuchung und beide Beiträge dürften sich wechselseitig in dem Grundanliegen unterstützen, den Beschluss als einen eigenständigen Rechtsakt zu erfassen, der nicht mit der Gesamtheit der Stimmabgaben gleichzusetzen ist.

132 Man wird dem Beschluss den Rechtsgeschäftscharakter heute jedenfalls nicht mehr deshalb absprechen, weil er keine Rechtswirkung (nach außen) hervorbringe; nach der überholten Lehre von u.a. P. Eltzbacher (Die Handlungsfähigkeit nach dt. bürgerl. Recht, 1. Bd., 1903, S. 168, 171) sollte der Beschluss nur eine juristische Tatsache sein, an die Organe oder Vertreter des Verbandes anzuknüpfen haben, wenn sie rechtsgeschäftlich für den Verband handeln. Zum Verhältnis von Beschluss und Ausführungsakt aus heutiger Sicht s. Jacoby (o. Fn. 11), S. 421 ff.

133 Es sei daran erinnert, dass die von Tubr'sche Vorstellung vom mehrseitigen, kraft Mehrheitsprinzip verbindlichen Rechtsgeschäft für einfache Mehrheitsentscheidungen, z.B. von Bruchteilseigentümern, brauchbar bleibt; oben II. 